\title{
Dysregulation of autophagy and stress granule-related proteins in stress-driven Tau pathology
}

\author{
Joana Margarida Silva ${ }^{1,2} \cdot$ Sara Rodrigues ${ }^{1,2} \cdot$ Belém Sampaio-Marques $^{1,2}$ • Patrícia Gomes ${ }^{1,2}$ • \\ Andreia Neves-Carvalho ${ }^{1,2} \cdot$ Chrysoula Dioli $\mathbb{D}^{1,2} \cdot$ Carina Soares-Cunha ${ }^{1,2} \cdot$ Brandon F Mazuik $^{3}$. \\ Akihiko Takashima $^{4} \cdot$ Paula Ludovico ${ }^{1,2} \cdot$ Benjamin Wolozin $\mathbb{1}^{3} \cdot$ Nuno Sousa ${ }^{1,2} \cdot$ Ioannis Sotiropoulos ${ }^{1,2}$
}

Received: 25 September 2017 / Revised: 1 June 2018 / Accepted: 4 June 2018 / Published online: 15 November 2018

(c) ADMC Associazione Differenziamento e Morte Cellulare 2018

\begin{abstract}
Imbalance of neuronal proteostasis associated with misfolding and aggregation of Tau protein is a common neurodegenerative feature in Alzheimer's disease (AD) and other Tauopathies. Consistent with suggestions that lifetime stress may be an important AD precipitating factor, we previously reported that environmental stress and high glucocorticoid (GC) levels induce accumulation of aggregated Tau; however, the molecular mechanisms for such process remain unclear. Herein, we monitor a novel interplay between RNA-binding proteins (RBPs) and autophagic machinery in the underlying mechanisms through which chronic stress and high GC levels impact on Tau proteostasis precipitating Tau aggregation. Using molecular, pharmacological and behavioral analysis, we demonstrate that chronic stress and high GC trigger mTORdependent inhibition of autophagy, leading to accumulation of Tau aggregates and cell death in P301L-Tau expressing mice and cells. In parallel, we found that environmental stress and GC disturb cellular homeostasis and trigger the insoluble accumulation of different RBPs, such as PABP, G3BP1, TIA-1, and FUS, shown to form stress granules (SGs) and Tau aggregation. Interestingly, an mTOR-driven pharmacological stimulation of autophagy attenuates the GC-driven accumulation of Tau and SG-related proteins as well as the related cell death, suggesting a critical interface between autophagy and the response of the SG-related protein in the neurodegenerative potential of chronic stress and GC. These studies provide novel insights into the RNA-protein intracellular signaling regulating the precipitating role of environmental stress and GC on Tau-driven brain pathology.
\end{abstract}

Edited by D. Rubinsztein

Electronic supplementary material The online version of this article (https://doi.org/10.1038/s41418-018-0217-1) contains supplementary material, which is available to authorized users.

$\triangle$ Ioannis Sotiropoulos

ioannis@med.uminho.pt

1 Life and Health Sciences Research Institute (ICVS), Medical School, University of Minho, Campus Gualtar, 4710-057 Braga, Portugal

2 ICVS/3B's_-PT Government Associate Laboratory, Braga/ Guimarães, Portugal

3 Department of Pharmacology \& Experimental Therapeutics, School of Medicine, Boston University, MA 02118 Boston, USA

4 Department of Life Science, Faculty of Science, Gakushuin University, 171-8588 Tokyo, Japan

\section{Introduction}

Alzheimer's disease (AD) is a multifactorial neurodegenerative disorder with a complex pathophysiology and still undefined initiators. Several risk factors have been associated with $\mathrm{AD}$ pathology, with recent evidence supporting a detrimental role of lifetime stress [1-3]. Clinical studies relate distress, high cortisol levels and dysfunction of hypothalamus-pituitary-adrenal (HPA) axis with poor memory scores and earlier disease onset in AD patients highlighting the potential implication of chronic stress and glucocorticoids (GC) in the pathogenesis and/or progression of the disorder [4-6]. In line with the above clinical evidence, experimental studies have shown that chronic stress and exposure to high-GC levels trigger Tau hyperphosphorylation and malfunction leading to its accumulation, formation of neurotoxic Tau aggregates and AD pathology $[1,7,8]$. Despite our little knowledge about the molecular mechanisms that underpin stress-driven pathology, 
experimental evidence suggests that stress/GC reduces Tau turnover [9], suggesting that stress/GC impact on the chaperones and proteases that regulate Tau levels [8].

Impaired proteostasis is thought to lead to the accumulation of misfolded and aggregated proteins, causing a corresponding increase in neuronal vulnerability and neurodegeneration. One sign of impaired proteostasis is the massive accumulation of autophagic vacuoles and proteins in the autophagic-lysosomal pathway which occurs in brains of AD cases [10, 11]. Divided in different steps, e.g., initiation, elongation, and maturation, the (macro)autophagy process critically involves several evolutionarily conserved molecules such as the microtubule-associated protein light chain 3 (LC3), autophagy receptor p62 and mammalian target of rapamycin (mTOR) [12]; the latter has been suggested as therapeutic target against pathological aggregation of Tau and related AD neurotoxicity [13, 14]. Moreover, inhibition of autophagic-lysosome pathway is also shown to impair the degradation and dynamics of stress granules (SGs) [15]. SGs are cytoplasmic complexes containing mRNAs and different RNA-binding proteins (RBPs) such as the SG-associated $\mathrm{T}$ cell intracellular antigen 1 (TIA-1), poly(A)-binding protein (PABP), fused in sarcoma protein (FUS), and the stress granule assembly factor, RasGTPase-activating protein binding protein 1 (G3BP1) [16]. Several SG-associated RBPs have been associated with neurodegenerative diseases with a close link being recently identified between the SGs core-nucleating protein TIA-1 and Tau. Specifically, TIA-1 is shown to bind to Tau contributing to its aggregation and Tau-related neurodegeneration and toxicity found in $\mathrm{AD}$ and other Tauopathies [17-19]. Based on the above, we hereby monitor the role and potential interplay between autophagy, SG-related proteins and Tau aggregation in stress-driven brain pathology.

\section{Methods and materials}

\section{Animals and stress protocol}

6-8-month-old P301L-Tau transgenic mice, expressing mutated (P301L) human Tau under the CAMKII promoter ( $N=7-9$ per group) were used in this study [20]. All experiments were conducted in accordance with the Portuguese national authority for animal experimentation, Direcção Geral de Veterinária (ID: DGV9457). Animals were kept and handled in accordance with the guidelines for the care and handling of laboratory animals in the Directive 2010/63/EU of the European Parliament and Council. Mice were housed in groups of four to five per cage under standard environmental conditions (lights on from 8 a.m. [ZT0] to 8 p.m. [ZT12]; room temperature $22{ }^{\circ} \mathrm{C}$; relative humidity of $55 \%$, ad libitum access to food and water). Animals were subjected to chronic unpredictable stress protocol over a period of 4 weeks before the behavioral testing. Experiments were replicated three times (7-9 mice per group for each experimental replicate). The stress protocol consists of different stressors, such as overcrowding, rocking platform, restrain and hair dryer (one stressor perday) that were chosen in a random order to prevent habituation. Stress efficacy was monitored by measurements of daytime serum corticosterone levels (monitored by a radioimmunoassay kit from ICN, Costa Mesa, CA) and body weight. All stressed animals showed significant elevations in daytime serum corticosterone levels $(p<0.05)$ and net loss of body weight $(p<0.05)$ reflecting the stress efficacy (see Fig. 1).

\section{Behavior testing}

Open field $(O F)$ test was conducted in a square arena $(43.2 \mathrm{~cm})$ with transparent acrylic walls and white floor (Med Associates Inc., St. Albans, VT, USA). Mice were placed in the center of the arena and their movement was automatically monitored over a period of $5 \mathrm{~min}$ with the aid of two 16-beam infrared arrays. Time spent in the center of the arena was used as an index of anxious behavior. Total distance traveled was used as an indicator of locomotor activity.

Elevated-plus maze (EPM) test was used to access anxious behavior. Briefly, animals were placed in the center of the EPM apparatus and entries as well as time spent in open and closed arms were measured for $7 \mathrm{~min}$ as previously described [21]. Data were collected using a chargecoupled device (CCD) camera by the use of NIH Image program (http://rsb.info.nih.gov/nih-image/) and were analyzed using customized software based on Matlab (version 7.2, Mathworks Co. Ltd., CA) with image analysis tool box (Mathworks Co. Ltd., CA).

$Y$-maze test was used to assess prefrontal cortex (PFC)dependent memory based on spontaneous alternation task. Briefly, animals were placed in the center of the Y-maze apparatus $(33 \mathrm{~cm} \times 7 \mathrm{~cm} \times 15 \mathrm{~cm})$ and allowed to freely move for $8 \mathrm{~min}$. The number and order of arm entries was recorded. Spontaneous alternations were calculated as the ratio of number of triads (sequence of three consecutive arm entries) and total arm entries.

Reversal learning test was performed in a swimming circular pool $(1 \mathrm{~m}$ diameter $)$ filled with water $\left(24^{\circ} \mathrm{C}\right)$ made opaque with a white bio-safe dye. The cylinder contained a slightly submerged transparent escape platform and placed in a room with landmark (reference) objects. Learning trials (3 trials/day; 60-s trial period) start by gently placing mice on the water surface close to the cylinder wall. After subjected to probe test, animals 
a

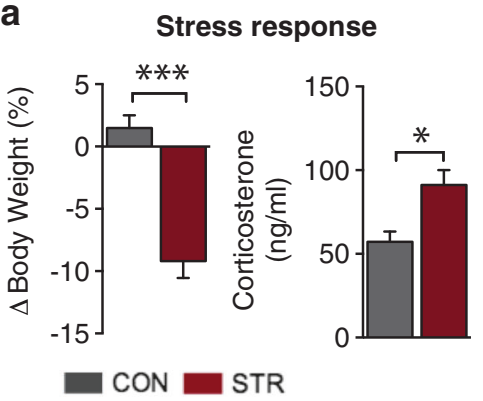

b

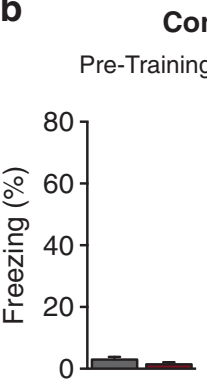

Contextual fear conditioning

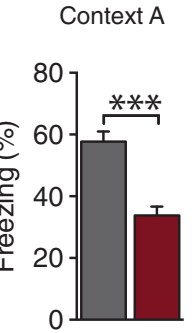

C

\section{Reversal}

learning
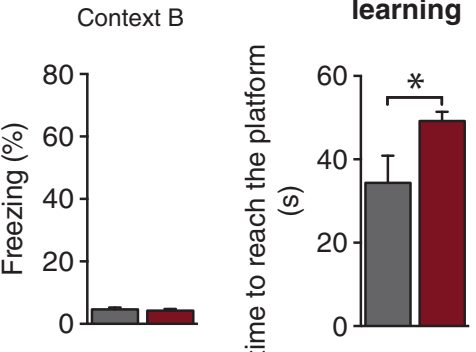

e

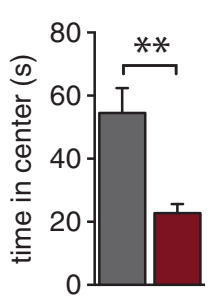

Open field

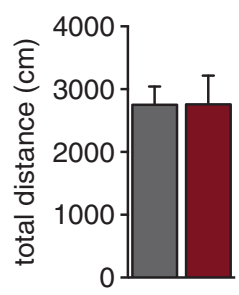

f Elevated-plus maze

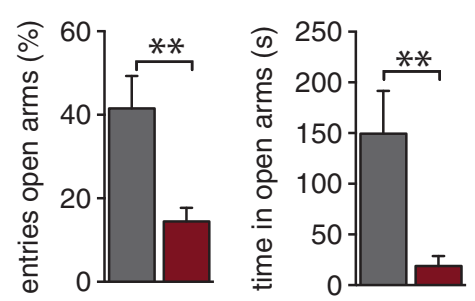

g

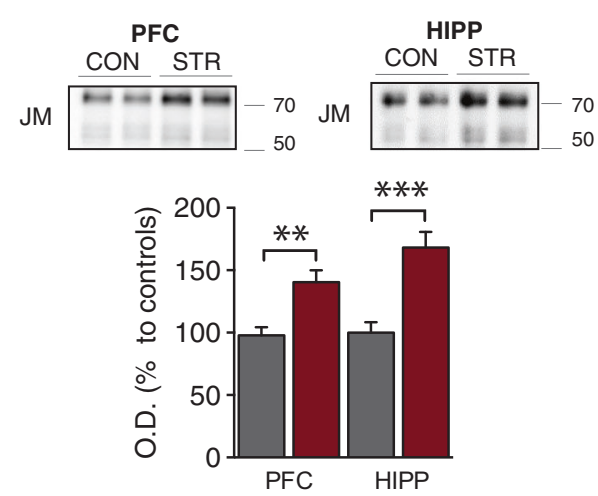

h h Cell density

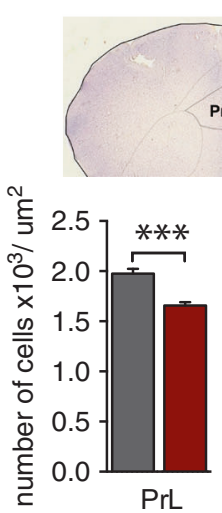

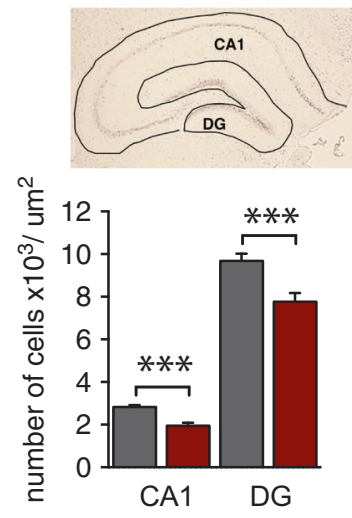

Fig. 1 Chronic stress evokes accumulation of neurotoxic Tau aggregates causing cognitive and emotional deficits in P301L-Tau Tg mice. a P301L-Tg mice exhibited reduced body weight $(p=0.005)$ and increased corticosterone $(p<0.001)$ levels after chronic environmental stress. b In contrast to pre-training session $(p=0.171)$, stressed animals exhibited a significant decrease in percentage of freezing time in test section (context A) of CFC in comparison to control animals indicating hippocampus-dependent associative memory impairment ( $p$ $<0.001$ ); note that both animal groups exhibit similar freezing levels in context $\mathrm{B}$ which is not associated with the adverse stimuli animals received in context A $(p=0.640)$. $\mathbf{c}$ Chronic stress increased the time that animals swum to reach the new (opposite) place of the escaping platform indicating PFC-dependent deficits of behavioral flexibility ( $p$ $=0.046)$. $\mathbf{d}$ Stress also reduced percentage of spontaneous alternations

were tested (4 trials) for reversal learning task where the platform was moved to the opposite quadrant of swimming pool. Swim path of each animal was recorded by a CCD camera and analyzed using customized software based on Matlab (version 7.2, Mathworks Co. Ltd., CA) with an image analysis tool box (Mathworks) $[20,22]$. in the arms of a Y-maze as compared with control animals pointing to deficits of working memory $(p=0.007)$. e-f Whereas no different in total distance traveled by animals in OF apparatus $(p=0.988)$, stressed animals exhibited a decrease in time spent in the center of the $\mathrm{OF}$ arena $(p=0.003)$ (e) followed by reduced time $(p=0.004)$ and entries $(p=0.001)$ spend in the open arms of EPM apparatus $(\mathbf{f})$; these behavioral parameters suggest increased levels of anxiety in stressed animals compared with controls. $\mathbf{g}-\mathbf{h}$ Chronic stress elevated the levels of sarkosyl-insoluble Tau in both hippocampus and PFC of P301LTau mice (Hipp: $p=0.028$; PFC: $p<0.001)(\mathbf{g})$; an effect that was accompanied by decreased cell density in PFC (prelimbic cortex; PrL: $p<0.001$ ) and hippocampus (DG: $p<0.001$; CA1: $p<0.001)(\mathbf{h})$. All numeric data represent mean \pm SEM, ${ }^{*} p<0.05$; ${ }^{* *} p<0.01 ;{ }^{* * *} p<0.001$

Contextual fear conditioning (CFC) test was conducted in CFC sound- and light-proof chambers (Med Associates, St. Albans, VT). On day 1, mice were placed in the conditioning white chamber (Context A) and received three pairings of light (20 s) and a co-terminating electrical shock ( $2 \mathrm{~s}, 0.5 \mathrm{~mA})$. The chambers were cleaned with $10 \%$ ethanol between animal trials. On day 2 , animals were placed in the 
same context (context A) in the absence of the light-shock stimuli. After it, the animals returned to their home cage. Two hours later, the animals were placed in a new context (context B) for 3 min. The context B trial was different from context A in several ways: (i) the floor and walls of the chamber were covered by black plastic inserts; (ii) the chamber was scented with vanilla; (iii) the chamber ventilation fan was turned on; (iv) the experimenter wore a different style and color of gloves, mask and lab coat; (v) mice were kept in a different holding room before testing and transported in a different cage; and (vi) the lights of the experimental room were turned on. Mice behavior was recorded by $\mathrm{CCD}$ camera and freezing behavior was manually scored using Kinoscope software (http:// sourceforge.net/projects/kinoscope/).

\section{Biochemical fractionation and immunoblotting}

Hippocampus and medial PFC tissue were macrodissected and immediately stored at $-80^{\circ} \mathrm{C}$. For detecting insoluble Tau, the sarkosyl-based fractionation protocol was used as previously described [20, 23]. After homogenization in Tris- $\mathrm{HCl}$ buffer $(10 \mathrm{mM}$ Tris, $150 \mathrm{mM} \mathrm{NaCl}$ including protease and phosphatases inhibitors), lysates were centrifuged at $100,000 \mathrm{~g}$. The pellet was re-homogenized in salt/ sucrose buffer $(0.8 \mathrm{M} \mathrm{NaCl}, 10 \mathrm{mM}$ Tris-HCl, 1 EGTA, $\mathrm{pH}$ E7.4, 10\% sucrose solution including protease and phosphatases inhibitors). After addition of $10 \%$ Sarkosyl solution (Sigma, \#L-5125), incubation $\left(37^{\circ} \mathrm{C} ; 1 \mathrm{~h}\right)$ and centrifugation $(150,000 \mathrm{~g})$, the resulting pellet was analyzed as sarkosyl-insoluble fraction. As insoluble stress granules (SGs) cannot be detected in sarkosyl-insoluble fractions [18], we separated soluble and insoluble SGs using radioimmunoprecipitation assay (RIPA) buffer $(50 \mathrm{mM}$ Tris, $1 \% \mathrm{NP}-40,0.25 \%$ sodium deoxycholate, $150 \mathrm{mM} \mathrm{NaCl}$, $1 \mathrm{mM}$ EGTA, including protease and phosphatase inhibitors) as previously described [19]. Homogenates were layered onto $0.32 \mathrm{M}$ sucrose buffer $(0.32 \mathrm{M}$ sucrose, $10 \mathrm{mM}$ Tris- $\mathrm{HCl}, 0.8 \mathrm{M} \mathrm{NaCl}, 1 \mathrm{mM}$ EGTA) and were centrifuged $\left(20,000 \mathrm{~g}, 10 \mathrm{~min}, 4^{\circ} \mathrm{C}\right)$. The supernatants were ultracentrifuge at $112,000 \mathrm{~g}\left(60 \mathrm{~min} ; 4^{\circ} \mathrm{C}\right)$. The supernatant (soluble fraction) was collected and stored at $-20^{\circ} \mathrm{C}$. The remaining pellet was dissolved in $1 \%$ sodium dodecyl sulfate (SDS)/RIPA buffer and centrifuged at $112,000 \mathrm{~g}$ (60 min; $4{ }^{\circ} \mathrm{C} ; 2$ times) followed by $1 \%$ SDS/TBS buffer and ultracentrifugation $\left(60 \mathrm{~min} ; 20^{\circ} \mathrm{C}\right)$. The final pellet was dissolved in $70 \%$ formic acid and centrifuged at $20,000 \mathrm{~g}$ $\left(10 \mathrm{~min} ; 20^{\circ} \mathrm{C}\right)$. The supernatant was concentrated in speed vacuum and the resulting pellet dissolved in sample buffer and neutralized with $1.5 \mathrm{M}$ Tris- $\mathrm{HCl}$ providing the insoluble SG fraction.

The above samples were electrophoresed using SDS-PAGE gels (17\%-acrylamide gel for LC3II detection,
$10 \%$-acrylamide for the remaining proteins) and semi-dry transferred onto nitrocellulose membranes (Trans-Blot Turbo blotting system, BIORAD). Membranes were blocked with 5\% non-fat dry milk in TBS-T buffer and then, incubated with the following antibodies: actin (1:2500; ABCAM, \#ab8224), LC3 (1:1000; Novus Biologicals, \#100-233), SQMTS1/p62 (1:1000; Novus Biologicals, \#H00008878-M01), S6K total (1:750; Cell Signaling, \#2708), p-S6K (1:750; Cell Signaling, \#9205), p38 total (1:750; Cell Signaling, \#9212) and p-p38 (1:750; Cell Signaling,\#4511); eIF4E (1:500, Santa Cruz, \#sc-9976), mTOR total (1:500; abcam \#32028), p-Ser2448-mTOR (1:200; Cell Signaling, \#5536), Tau5 (1:2000; ABCAM, \#ab80579), JM (1:1000; kindly gift by Dr. A. Takashima, Japan, recognizes human Tau), TLS/FUS (1:500; ABCAM, \#84078), EWRS1 (1:1000; ABCAM, \#133288), DDX5 (1:1000; ABCAM, \#21696), TIA-1 (1:500; ABCAM, \#40693), PABP (1:500, ABCAM \#21060), tubulin (1:5000, SIGMA \#9026), Ac-tubulin (1:1000, ABCAM \#24610), Cortactin (1:250, ABCAM \#81208), Ac-cortactin (1:10000, Millipore \#09881), and HDAC6 (1:100, ABCAM \#1440). After incubation with appropriate secondary antibody, antigens were revealed by ECL (Clarity, Bio-Rad), and signal quantification was achieved using a ChemiDoc instrument and ImageLab software (Bio-Rad). All values were normalized and expressed as a percentage of control values.

\section{Immunofluorescence and stereological analysis}

As previously described [20], deeply anesthetized animals were transcardially perfused with saline and PFA (4\%). After postfixation, brains were placed in $30 \%$ sucrose and sectioned using vibratome. Sections were exposed to antigen retrieval by citrate buffer, followed by $0.3 \%$ Triton $\mathrm{X}-100$ before blocking with solution (5\% BSA in TBSTriton X-100 $(0.25 \%)+5 \%$ fetal bovine serum (FBS)) for $30 \mathrm{~min}$ (RT). Then, incubation with appropriate primary antibody: LC3 (1:100; Novus Biologicals), SQMTS1(p62) (1:500; Novus Biologicals), TIA-1 (1:200; ABCAM, \#40693), PABP (1:100, mouseAb, SantaCruz \#166381), PABP (1:200, rabbitAb, ABCAM \#21060), and PHF1 (1:100, kindly provided by Dr. Peter Davies), followed by incubation with the appropriate secondary antibodies and DAPI staining (1:1000; $10 \mathrm{~min})$. For LC3 and p62, stained cells were counted and densities were quantified and normalized for number of cells using the Olympus BX 51 stereological microscope and the Visiopharma integrator system software. Neuronal densities of hippocampal and PFC areas (DG, CA1, and PrL) were stereologically estimated by counting neurons in cresyl-violet stained serial coronal brain sections, using Neurolucida software (MBF Bioscience, Williston, VT) as previously described [24, 25]. 


\section{Human AD brain tissue}

Temporal cortex tissue from human brain was used for the immunohistochemical studies. Human tissue was sectioned at $20 \mu \mathrm{m}$ on a cryostat and stained as free floating sections. To quench autofluorescence, sections were photobleached under a 1500 lumen white LED bulb for a minimum of $72 \mathrm{~h}$ at $4{ }^{\circ} \mathrm{C}$ while suspended in PBS [26]. Sections were then washed in PBS followed by TBS with $0.25 \%$ Triton-X incubation. Samples were then incubated in $1 \% \mathrm{w} / \mathrm{v}$ sodium borohydride (NaBH4; Sigma-Aldrich Cat\#452882-25 G) in PBS for $45 \mathrm{~min}$ to quench aldehyde autofluorescence which results from the over-fixation of tissue. Then, citrate incubation was preformed at $95^{\circ} \mathrm{C}$ (Vector Cat\#H-3300), followed by blocking with donkey serum in PBS-T; primary antibody incubation with PABP (1:150, ABCAM \#21060), DDX6 (1:150, Bethyl Laboratories \#A300-460A), and pTau-CP13 (1:150, kindly provided by Dr. Peter Davies) was followed by incubation with the appropriate secondary. All imaging was done using a Zeiss LSM 700 confocal microscope.

\section{P301L-Tau-SH-SY5Y cells, treatments and analysis}

These studies used SH-SY5Y cells stably transfected with P301LhTau-EGFP (2N4R) (kind gift from Professor Juergen Gotz, University of Queensland, Australia). Cells were cultured in DMEM supplemented with $10 \%$ FBS, $1 \%$ Gmax and $1 \%$ antibiotic $\left(37{ }^{\circ} \mathrm{C}\right.$ and $5 \% \mathrm{CO}_{2}$; all reagents obtained by Invitrogen); for selection purposes, $3 \mu \mathrm{g} / \mathrm{mL}$ Blasticidin S hydrochloride (Sigma, \#15205) was added in the medium. For all experiments, cells were placed on gelatin-coated plates and differentiated for 6-7 days with differentiation medium [DMEM, 1\% FBS, 1\% antibiotics, $1 \%$ glutamax, $10^{-5} \mathrm{M}$ all-trans retinoic acid (Sigma)]. Each experiment has 3-4 biological replicates per condition, and experiments were repeated at least three times. Dexamethasone (DEX; Fortecortin ${ }^{\circ}$, Merck, Darmstadt, Germany) was used at a final concentration of $10^{-6} \mathrm{M}$ for $48 \mathrm{~h}$, as previously described [9]. For mTOR inhibition, Temsirolimus (CCI-779, LC Laboratories; $100 \mu \mathrm{M} ; 48 \mathrm{~h}$ ) was used; CCI-779 exhibits no toxicity at this concentration [27, 28]. Cell viability was assessed by MTS assay (CellTiter $96^{\circ}$, Promega, WI, USA) based on manufacturer instructions by the use of ELISA reader (BioRad); triplicates of each condition were used and experiment were repeated three times. For puromycin and cycloheximide treatment, cells were incubated with $5 \mu \mathrm{g} / \mathrm{ml}$ puromycin (Sigma \#P8833) or 10 $\mu \mathrm{g} / \mathrm{mL}$ cycloheximide (Millipore, \#CAS-66-81-9) $6 \mathrm{~h}$ before the end of the $48 \mathrm{~h}$ DEX incubation period. For Bafilomycine treatment, cells were incubated with
$10 \mathrm{nM}$ Bafilomycine A1(Bafilomycin A1 Santa Cruz \#CAS-88899-55-2) for $4 \mathrm{~h}$ for analysis of autophagy flux. For WB analysis, cell homogenates were prepared in RIPA buffer $(50 \mathrm{mM}$ Tris $\mathrm{HCl}, 2 \mathrm{mM}$ EDTA, $250 \mathrm{mM}$ $\mathrm{NaCl}, 10 \%$ glycerol, proteinase, and phosphatase). After sonication and centrifugation $\left(15 \mathrm{~min} ; 14,000 \mathrm{rpm} ; 4{ }^{\circ} \mathrm{C}\right)$, supernatant samples were analyzed by WB as described above. Total and sarkosyl-resistant Tau aggregates of cell homogenates were detected after sample microfiltration. Briefly, $60 \mu \mathrm{g}$ of samples were diluted in $200 \mu \mathrm{l}$ of PBS with or without $1 \%$ (wt/vol) Sarkosyl and boiled for $5 \mathrm{~min}$. Samples were filtered through a cellulose acetate membrane $(0.2 \mu \mathrm{m}$, Whatman), pre-equilibrated in PBS, using a Bio-Dot SF microfiltration apparatus (Bio-Rad). The membrane was washed twice with PBS before being probed with Tau-5 antibody (1:2500, Abcam) as described above. For intensity factor (IF) analysis, cells were cultured in gelatin-coated glass coverslips and fixed in 4\%PFA. After permeabilization with $0.1 \%$ TritonX-100/PBS, cells were incubated overnight with primary antibodies: LC3 (1:200; Novus Biologicals, \#100-233), SQMTS1(p62) (1:200; Novus Biologicals, \#H00008878-M01), Tau-5 (1:1000; ABCAM, \#ab80579), TLS/FUS (1:300; ABCAM, \#84078), G3BP (1:500; ProteinTech, \# 13057-2AP), TIA-1 (1:300; ABCAM, \#40693), and HDAC6 (1:200; ABCAM, \#ab1440). After appropriate fluorescenceconjugated secondary antibodies (RT, $30 \mathrm{~min}$ ) and DAPI staining, cells were analyzed by laser confocal microscopy (Zeiss LSM 510, Carl Zeiss Microimaging, Goettingen, Germany).

\section{Statistical analysis}

Numerical data is expressed as group mean \pm SEM. All data were evaluated by Student's $t$ test or one-way ANOVA (followed by post hoc Sidak test) when appropriate, using GraphPad 6.0; differences were considered to be significant if $p<0.05$.

\section{Results}

\section{Chronic stress triggers accumulation of neurotoxic Tau aggregates in hippocampus and prefrontal cortex of P301L-Tau Tg mice}

The hippocampus and the PFC are some of the brain areas most affected in $\mathrm{AD}$, exhibiting a characteristic accumulation of pathological, aggregated Tau (e.g., hyperphosphorylated, truncated, and misfolded); the accumulation of pathological Tau is closely correlated with cognitive impairment [29, 30]. Previous studies have shown that 
stress and GC trigger aberrant hyperphosphorylation, misfolding, and missorting of Tau [7, 9, 31, 32]. In the current study, we sought to clarify the impact of chronic stress on Tau aggregation mechanism(s) and resulting behavioral deficits. These studies used the P301L-Tau transgenic mice expressing 4R0N human Tau carrying the aggregationprone P301L-Tau mutation [20]; these mice were then subjected to chronic unpredictable stress. Stressed P301LTau animals exhibited a clear decrease in body-weight in comparison to control (nonstressed) animals (Fig. 1a). They also showed elevated levels of the stress hormone, corticosterone (Fig. 1a), providing confirmation of the stress protocol efficacy. To access hippocampus-dependent cognitive performance, CFC test was used. While both groups showed similar freezing levels in pre-training session of context A, stressed animals exhibited lower levels of freezing at the next day (Context A) compared to control animals indicating deficits of associated memory (Fig. 1b). Of note, the stress-driven difference disappeared when both groups where tested in another, non-associated with adverse stimulus, context (context B) (Fig. 1b). Furthermore, animals behavioral flexibility and working memory function were tested using the reversal learning and Y-maze test, respectively. During the reversal learning test, stressed animals exhibited an increase in the time to reach the escaping platform placed at the opposite quadrant in comparison to control animals suggesting cognitive deficits (Fig. 1c). In the Y-maze, stressed mice presented a decrease in the percentage of spontaneous alternations among different arms of the Y-maze apparatus (Fig. 1d), pointing toward a stress-driven impairment in working memory. As non-cognitive neuropsychiatric symptoms, such as anxiety, are frequently observed in $\mathrm{AD}$ [33], we also evaluated anxious behavior using OF and EPM. We found that chronic stress decreased the time that animals spent in the center of the OF arena (Fig. 1e) and reduced both time and entries in the open arms of EPM apparatus (Fig. 1f). Notably, stress did not cause any change in locomotion as assessed by total distance traveled in the OF apparatus (Fig. 1e). The above stress-driven behavioral deficits in P301L-Tau Tg animals were accompanied by increased levels of sarkosyl-insoluble Tau in both hippocampus and PFC of stressed animals as measured by Western blot analysis (Fig. 1g). These Tau aggregates are biochemically similar to those found in the neurofibrillary tangles that characterize AD and other Tauopathies [30]. Furthermore, P301L-Tau aggregates are shown to exhibit a major trigger of neurotoxicity in Tau pathology [20], which is in line with the reduction of cell density that stressed P301L-Tau animals exhibited in hippocampus (CA1 and DG) and PFC (prelimbic cortex; PrL) when compared to control P301LTau animals (Fig. 1h).

\section{Stress-driven inhibition of autophagy through mTOR activation}

A growing body of research has connected autophagy to neurodegenerative pathology while autophagic clearance has been suggested to exhibit selectivity for the degradation of Tau aggregates [11]. Accordingly, we have monitored autophagy focusing on LC3 and p62, the most widely used indicators of autophagic flux (Fig. 2a). Molecular analysis of hippocampus and PFC of P301L-Tau animals showed that chronic stress reduced the levels of LC3 and increased levels of p62 (Fig. 2b) in hippocampus and PFC; this was further confirmed by parallel shifts in the IF of LC3 and p62 (Fig. 2c-e). As many studies describe an essential role for mTOR in protein homeostasis through its involvement in the initiation of autophagic process [34], we assessed mTOR activity by analyzing mTOR and some of its downstream targets (Fig. 2f). We found that chronic stress increased the levels of phosphorylated mTOR and the stress-activated protein kinase, p38; the later is suggested to be involved in both Tau pathology and mTOR activation [35-37]. Furthermore, the phosphorylated levels of downsteam mTOR target, S6K was also elevated by stress while the protein levels of the downstream target, eIF4E were decreased by chronic stress (Fig. 2f). Altogether, the above findings suggest that exposure to chronic stress may inhibit autophagy, probably at the level of mTOR-mediated induction of autophagy (Fig. 2a).

\section{Glucocorticoid treatment mimic the stress-driven effect on aggregation-enhancing neurodegenerative cascades in vitro}

Despite the fact that the detrimental effects of chronic stress on neuronal structure and function are largely attributed to GC [38], and previous work highlights the role of glucocorticoid receptor (GR) in Tau malfunction and pathology [7, 9], other studies exclude GC and GR signaling from the effect of stress on Tau [39]. Thus, we next tested the impact of prolonged treatment with high GC levels using the synthetic glucocorticoid dexamethasone on a neuronal cell line expressing P301L-Tau tagged with GFP. We found that GC treatment $\left(10^{-6} \mathrm{M}\right.$, $48 \mathrm{~h}$ ) caused increase of GFP-labeled P301L-Tau protein as well as total Tau levels (Fig. 3b, c) in parallel to decreased cell viability (Fig. 3a). In addition, WB analysis revealed that GC impacted both on wild-type (human) Tau and exogenously expressed (P301L-Tau) human Tau protein as well as insoluble levels of Tau (Fig. 3c, d). Using both IF and WB analysis, we found that autophagic markers such as LC3 and p62 are also affected by GC treatment. In line with our in vivo findings 

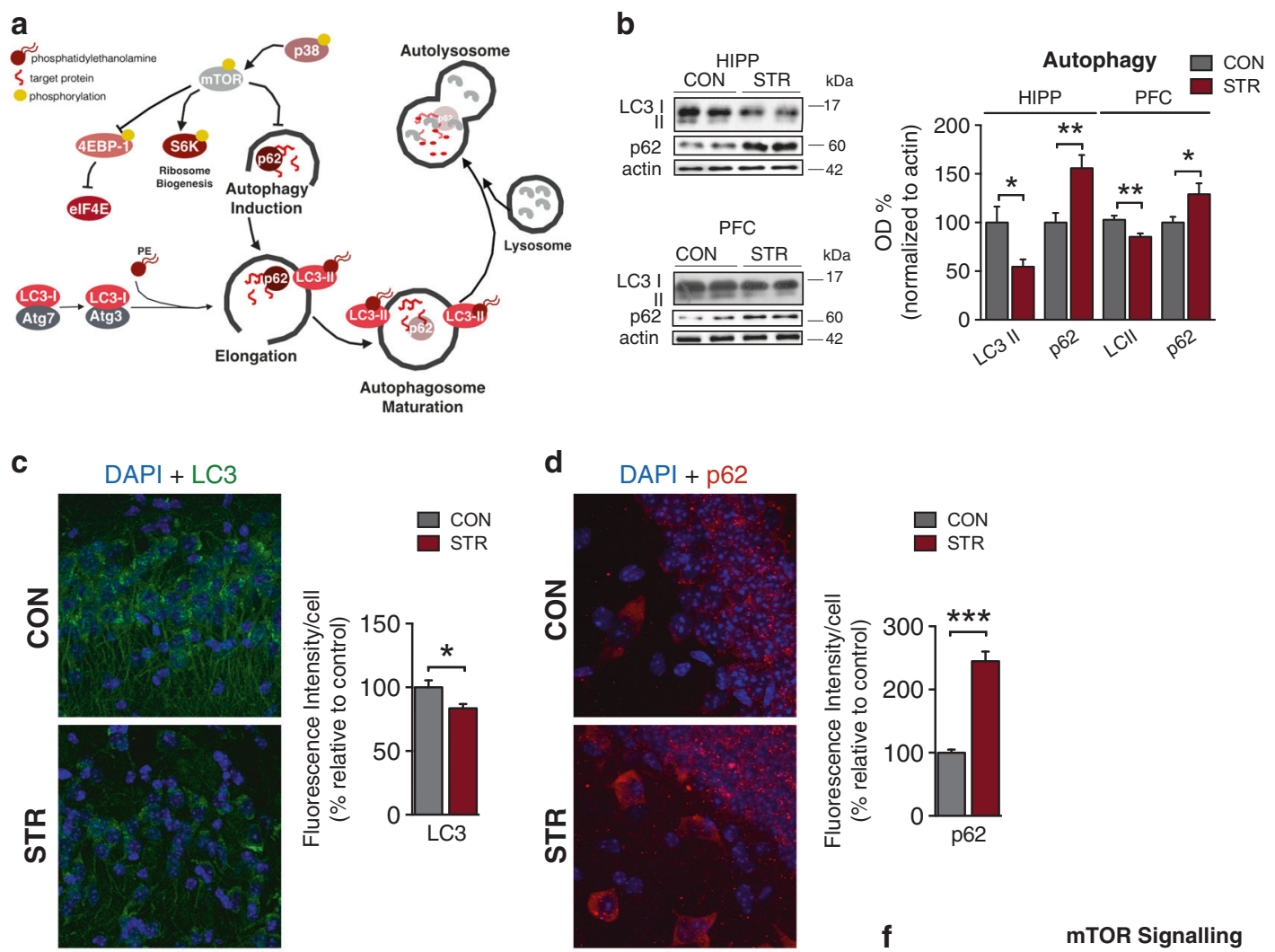

d

d $\quad \mathrm{DAPI}+\mathrm{p} 62$

e

DAPI + LC3
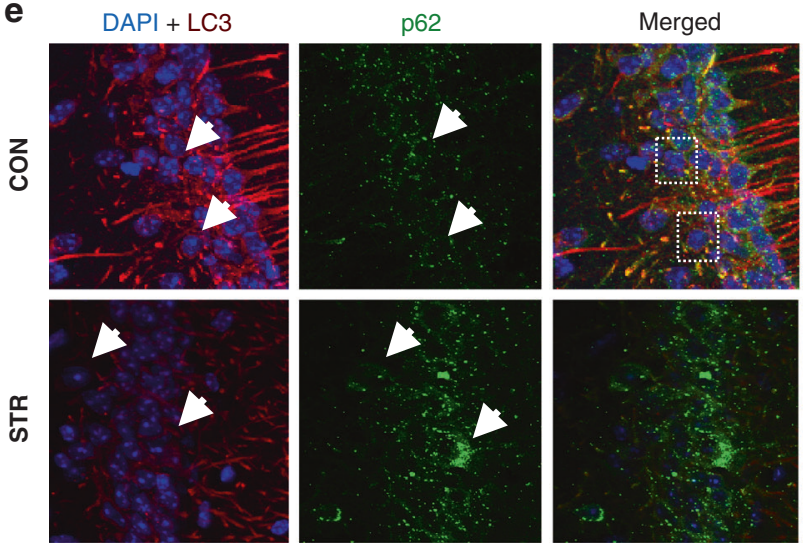

Fig. 2 Prolonged exposure to environmental stress inhibits autophagy in a mTOR-dependent manner. a Schematic representation of autophagy highlighting the role of mTOR, LC3, and p62 in this cellular process. b-e Stressed animals exhibited reduced LC3 (PFC: $p=0.003$; HIPP: $p=0.026$ ) and increased p62 (PFC: $p=0.029$; HIPP: $p=$ $0.004)$ protein levels as assessed by WB analysis (b); these findings were confirmed by corresponding changes in LC $3(p=0.044)$ and p62 $(p=0.044)$ fluorescence intensity/cell number $(\mathbf{c}-\mathbf{d})$ an decrease in

in P301L-Tau mice, GC treatment of P301L-Tau cells led to an increase in p62 levels accompanied by a reduction in LC3II measured by WB analysis (Fig. 3e). Accordingly, we also observed a decrease in $\mathrm{LC}^{+}$puncta after $\mathrm{GC}$ exposure (Fig. 3f). Furthermore, in another experiment monitoring autophagic flux by bafilomycin A1, a known inhibitor of the late phase of autophagy, GC led to accumulation of p62 in parallel to a decrease of LC3II levels compatible with an inhibition of autophagy (Supplementay Fig. S1). Altogether, the above results indicate that GC induced a blockage of autophagic clearance and accumulation and aggregation of Tau. 

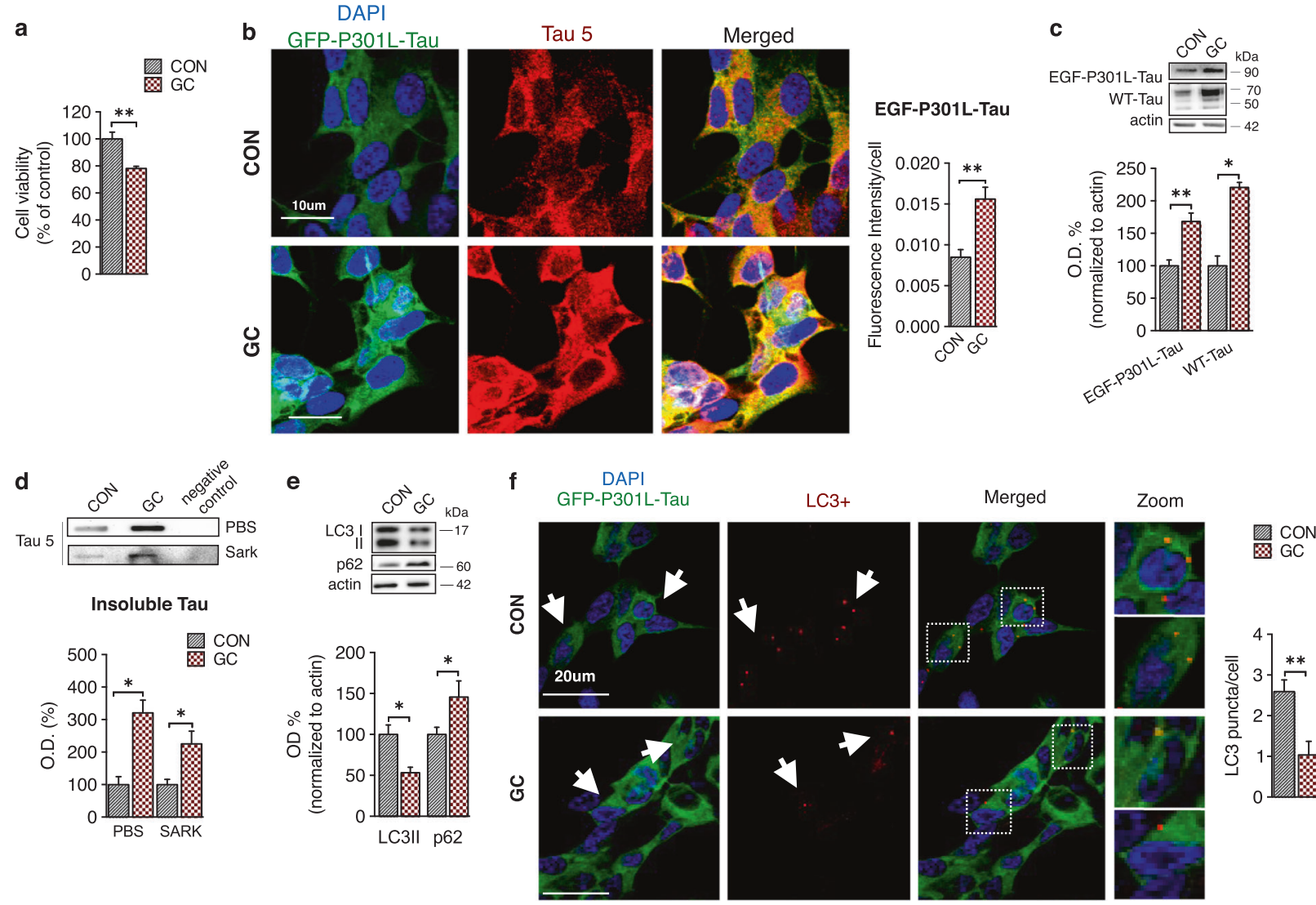

Fig. 3 Exposure to glucocorticoids causes Tau accumulation and autophagy inhibition in vitro. a GC treatment $\left(10^{-6} \mathrm{M} ; 48 \mathrm{~h}\right)$ of EGFP301L-hTau SH-SY5Y cells decreased cell viability $(p=0.004)$. b-d Glucocorticoid (GC) treatment triggered cytoplasmic accumulation of exogenously expressed mutated human Tau (EGF-P301L-Tau) and endogenous human Tau (WT-Tau) as assessed by IF (f) $(p=0.044)(\mathbf{b})$ and WB analysis (c) (WT-Tau: $p<0.001$; EGF-P301L-

Tau: $p<0.001)$, and lead to increased levels of insoluble Tau aggregates $(p=0.042)(\mathbf{d})$. e-f GC decreased LC3II $(p=0.013)$ protein levels with parallel increase of p62 $(p=0.039)(\mathbf{e})$; immunofluorescence analysis confirmed the GC-induced reduction in LC3 $(p=0.004)$ puncta $(\mathbf{f})$. All numeric data represent mean \pm SEM, ${ }^{*} p<0.05 ;{ }^{* *} p<0.01 ;{ }^{* * *} p<0.001$

\section{Chronic stress and glucocorticoids trigger accumulation of stress granule-related proteins}

The cellular stress response under pathological conditions includes the translational stress response, which results in the dysregulation of RBPs and consequent SG formation. These SGs have also recently been shown to stimulate the accumulation of aggregated Tau [16, 18]. As inhibition of autophagic-lysosome pathway was recently shown to impair the degradation and dynamics of SGs [15], we next monitored different SG-related proteins as well as their insoluble accumulation in P301L-Tau animals under control and stressful conditions.

Our analysis showed the presence of different RBPs and SGs markers in soluble and insoluble fractions of homogenates of P301L-Tau mice under control (nonstressed) conditions (Fig. 4), which is in line with previous studies that show the association of many SG-related proteins with pathological Tau [18, 19]. In addition, we observed that chronic stress significantly elevated cytoplasmic localization of TIA-1, and also increased the soluble levels of RBPs proteins TIA-1, DDX5, EWRS1, and TLS/FUS (Fig. 4a); similar increases of insoluble levels of TLS/FUS, DDX5, and PABP were observed with chronic stress (Fig. 4b). Notably, TIA-1 was not detected in the insoluble fraction in contrast with previous work that did not utilize a stress model [18, 40]. Confirming the above results, immunofluorescence staining demonstrated that chronic stress increased total and cytoplasmic TIA-1 staining in hippocampus together with a striking increase in cytoplasmic co-localization with Tau phosphorylated at amino acids 396 and 404, detected with the PHF1 antibody (Fig. 4c). We also observed an increase in PABP staining in hippocampus after stress, that co-localizes with PHF-1 pTau (Fig. 4d) and TIA-1 (Fig. 4e). RNA binding proteins, such as DDX6 and PABP, diffuse around p-Tau inclusions in human $\mathrm{AD}$ brain (Fig. 4f), although in the human brain the RBPs appear adjacent to the inclusion, which reflects increasing exclusion as the Tau pathology consolidates; similar findings are observed in late stage mouse models of 
a

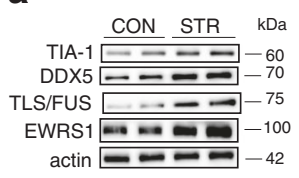

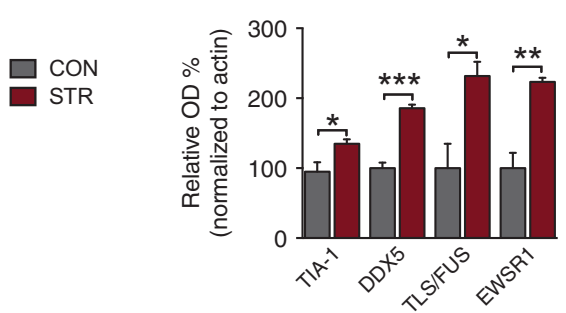

b

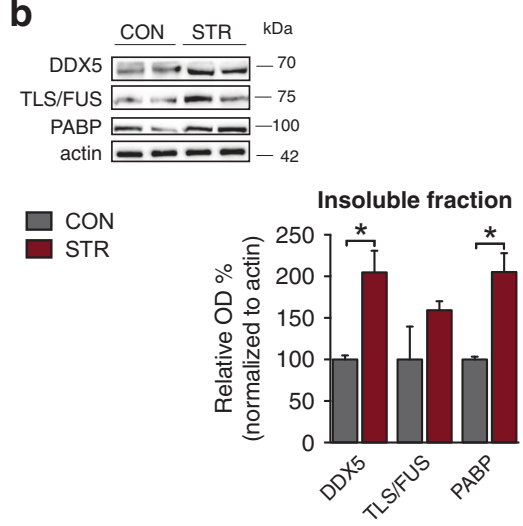

C

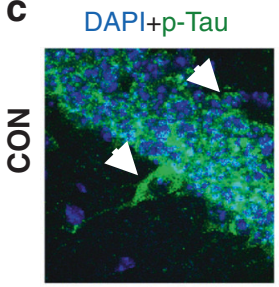

辇

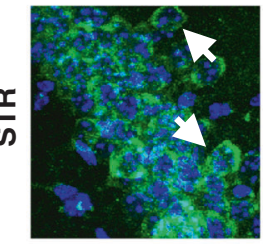

d
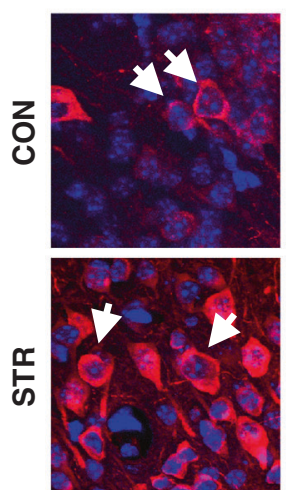
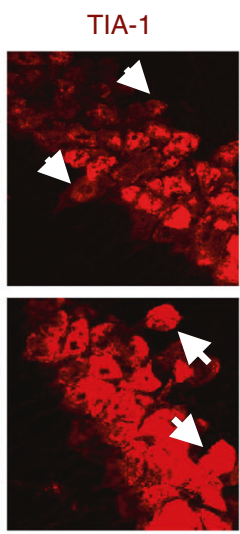

p-Tau
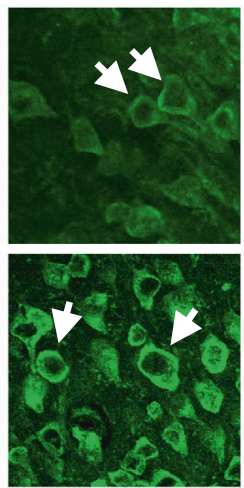

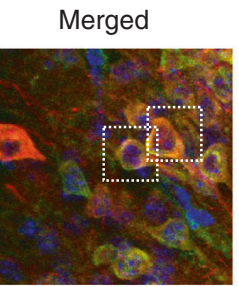

Zoom
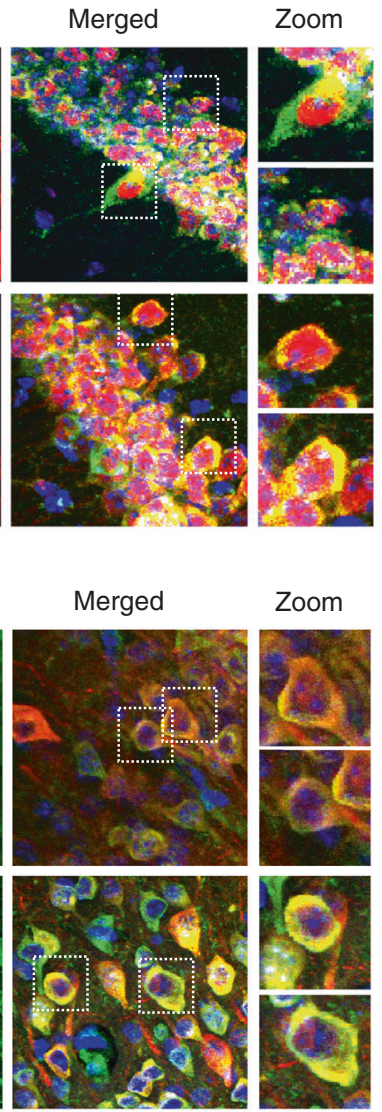
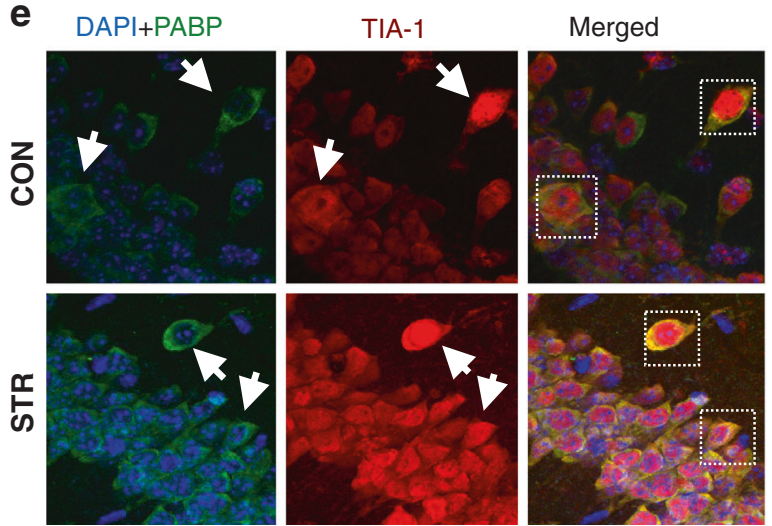

Zoom

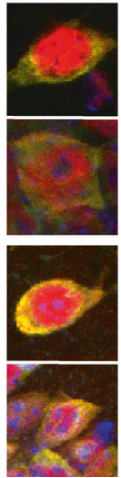

f

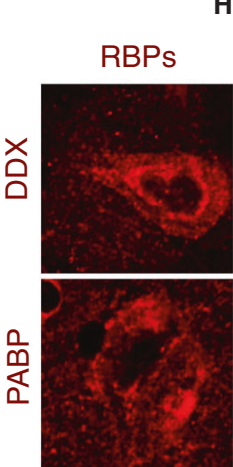

Human AD Brain
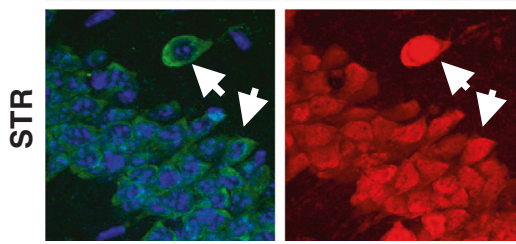

Fig. 4 Chronic stress evokes dysregulation of RBPs and their insoluble accumulation in P301L-Tau Tg mice. a, b Chronic stress triggered an increase in the protein levels of several RBPs and SG markers in soluble fraction [TIA-1 $(p=0.013)$, TLS/FUS $(p=0.017)$, DDX5 ( $p$ $<0.001)$ and EWRS1 $(p=0.005)](\mathbf{a})$, as well in insoluble fraction of P301L-Tau mice [DDX5 $(p=0.02)$ and PABP $(p=0.011)](\mathbf{b}) . \mathbf{c}, \mathbf{d}$ Chronic stress causes the cytoplasmic accumulation of the SG marker TIA-1 in hippocampus (c) and the accumulation of PABP, a SG

tauopathy [19]. Thus, these findings suggest that chronic stress triggers the accumulation of different SG-related proteins which points toward a potential role for RBPs dysregulation in stress-driven Tau pathology.

marker (d), increasing their co-localization with p-Tau (PHF1). e Moreover, chronic stress leads to an increase co-localization of TIA-1 and PABP in perinuclear region of hippocampal neurons, indicating the presence of TIA-1 positive stress granules. $\mathbf{f}$ Immunofluroresecent analysis of PABP and DDX with p-Tau in human AD brain. All numeric data are represented as mean \pm SEM, ${ }^{*} p<0.05 ;{ }^{* *} p<0.01$; **** $p<0.001$

GC exposure in a cell line of SH-SY5Y cells stably expressing P301L-Tau also resulted in increased cytoplasmic levels and inclusions of different SG-related proteins such as TIA-1, TLS/FUS, DDX5, and G3BP (Fig. 5a). 
a
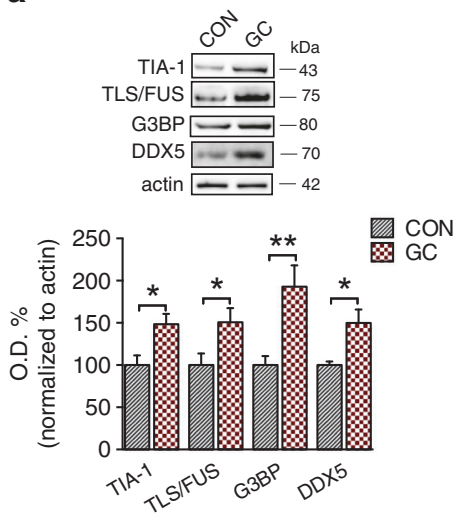

C
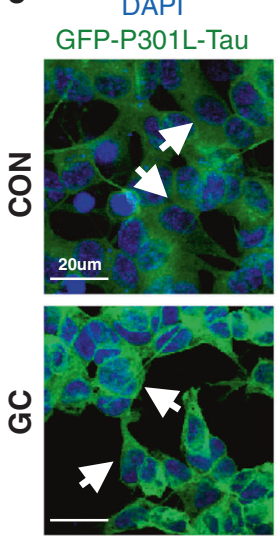

b
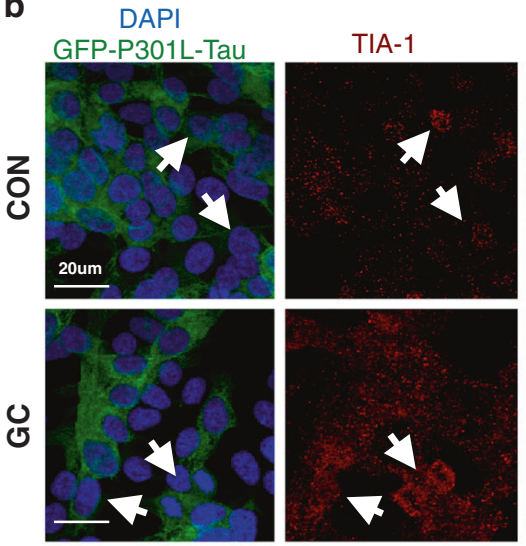

d
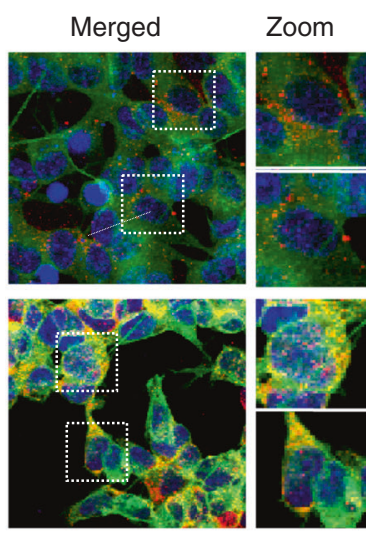
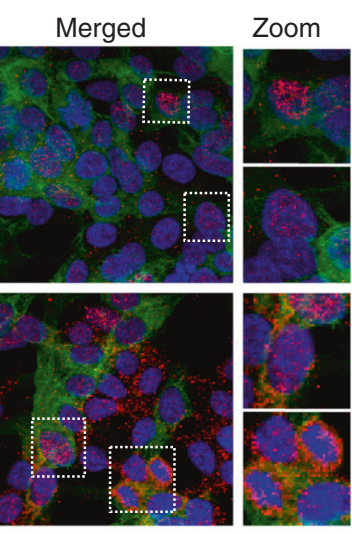

Fig. 5 Dysruption and insoluble accumulation of RBPs in GC-driven Tau accumulation. Treatment of EGF-P301L-hTau SH-SY5Y cells with GC $\left(10^{-6} \mathrm{M} ; 48 \mathrm{~h}\right)$ elevated the cytoplasmic levels of the RBPs, TIA-1 $(p=0.043)$, TLS/FUS $(p=0.034)$, G3BP $(p=0.004)$, and DDX5 $(p=0.038)$ as assessed by WB analysis. $\mathbf{b}, \mathbf{c}$ IF staining of TIA-1 (b) and G3BP (c) demonstrate that GC triggered their accumulation and cytoplasmic appearance in EGF-P301L-hTau SH-SY5Y

IF staining confirmed the GC-induced increased labeling of SG-related proteins TIA-1 and G3BP with increased TIA-1 reactivity and cytoplasmic translocation under GC exposure (Fig. 5b, c). For further monitoring the role of SG-related proteins in GC-driven Tau accumulation, we co-treated P301L-Tau cells with GC and either cycloheximide (CHX) or puromycin (PUR); two compounds that are protein synthesis inhibitors, but exhibit opposite effects on SG, promoting (PUR) or inhibiting (CHX) SGs assembly, respectively [19]. We found that GC and PUR co-treatment exacerbated the GC-induced increase of Tau (Fig. 5d), followed by increased levels of TIA-1 (Supplementary Fig. S2). In contrast, GC and CHX co-treatment partially attenuated GC-driven accumulation of Tau (Fig. 5e) suggesting a critical role for RBPs accumulation and the related SGs in the cellular mechanisms of GC-driven Tau accumulation.

cells. d Co-treatment of GC and puromycin (PUR), a well-known SG inducer, aggravate levels of EGF-P301L-Tau $(p=0.013)$ and WT-Tau $(\mathrm{p}=0.023)$ when compared to GC treatment. e While co-treament of GC and CHX (the later inhibits SG formation) blocked the GC-driven Tau increase $(p<0.001)$. All numeric data are represented as mean \pm SEM, ${ }^{*} p<0.05 ;{ }^{* * *} p<0.01 ;{ }^{* * *} p<0.001$

\section{Chronic stress and glucocorticoids impact on HDAC6 and its cytoplasmic downstream targets}

Importantly, SGs as well as different parts of autophagic process (e.g., autophagosome maturation) rely on microtubule-based networks and cytoskeletal machinery. Histone deacetylase 6 (HDAC6) regulates SGs and autophagy through its influence on different cytoskeletal molecules (e.g., tubulin, cortactin), and corresponding regulation of microtubule-dependent motility [41, 42]. Because HDAC6 is required for the consolidation of cellular complexes related to SGs and autophagy [41, 42], we proceeded to analyze the impact of GC on HDAC6 and its cytoskeletal targets. WB and IF analyses revealed that HDAC6 levels were increased by chronic stress and GC in P301L-Tau mice and cells (Fig. 6a, c, e), which was accompanied by a decrease in acetylation levels of the cytoskeletal targets of 
Fig. 6 Stress and glucocorticoids induce HDAC6 reducing the acetylation levels of its cytoplasmic targets. $\mathbf{a}, \mathbf{b}$ Chronic stress elevated HDAC6 levels $(p=0.004)(\mathbf{a})$ and reduced acetylation of tubulin $(p=0.016)$ and cortactin $(p=$ 0.048), two cytoskeletal targets of HDAC6, in P301L-Tau mice (b). c-e GC treatment increased HDAC6 $(p<0.001)$ as analyzed by WB (c) and elevated HDAC6 staining in P301LTau cells $(p=0.041)(\mathbf{e})$, in parallel with decreased levels of acetylated forms of tubulin $(p=$ $0.004)$ and cortactin $(p<0.001)$, two cytoskeletal targets of HDAC6 (d). All numeric data are represented as mean $\pm \mathrm{SEM}$, ${ }^{*} p<0.05 ;{ }^{* *} p<0.01 ;{ }^{* * *} p<$ 0.001 a

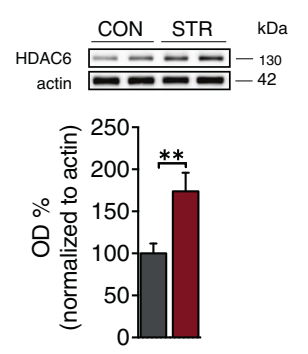

C

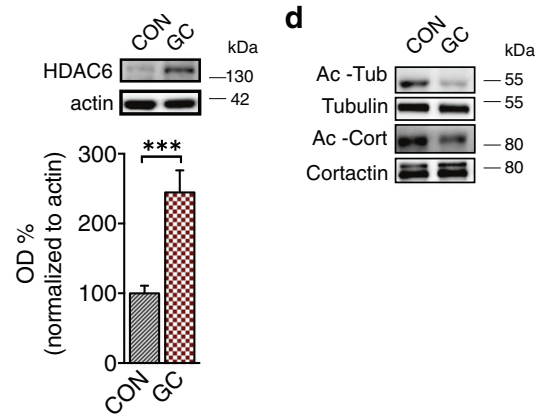

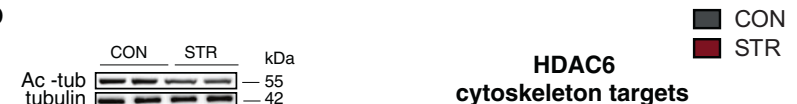

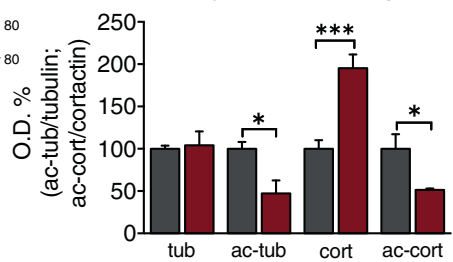

HDAC6
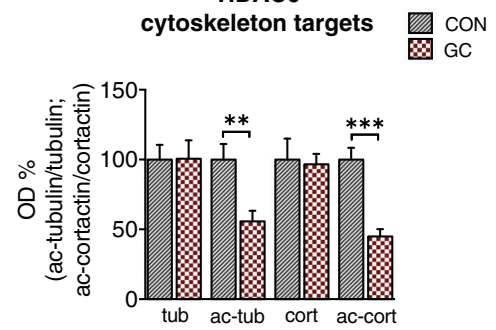

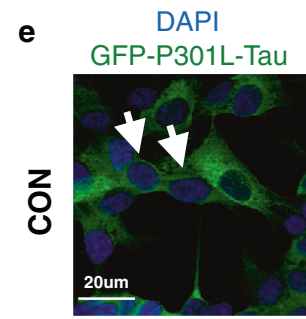

HDAC6

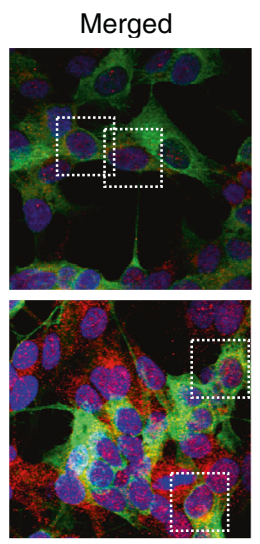

Zoom

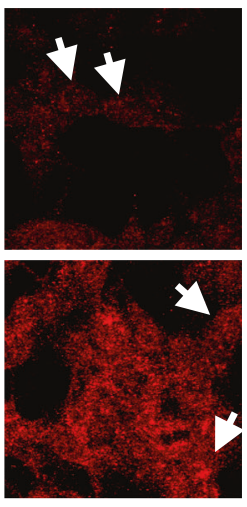

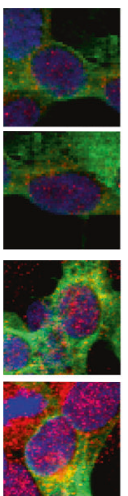

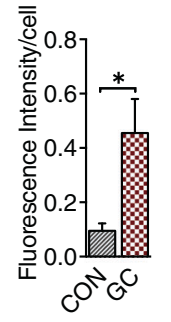

HDAC6, tubulin and cortactin (Fig. 6b, d). Matching with the above stress-driven deficits in autophagic and SGrelated RBPs, these data suggest a potential cytoplasmic role of HDAC6 in the stress/GC-driven cellular cascades related to imbalanced Tau proteostasis.

\section{Pharmacological stimulation of autophagy attenuates GC-driven neurotoxic cascades}

Mounting evidence supports mTOR as an important regulator of protein homeostasis [13], while the above in vivo data suggests that the inhibitory effect of stress on autophagic process is mTOR-related. Thus, we next clarified whether pharmacological inhibition of mTOR could protect against GC-driven Tau-related neurotoxicity. For that purpose, we used a rapamycin analog, Temsirolimus (CCI-779) shown to be safe and recently approved by the USA and European Drug authorities [43]. Co-treatment of CCI-779 with GC attenuated the reduced cell viability caused by GC (Fig. 7a) providing neuroprotection. Furthermore, we found that $\mathrm{GC}+\mathrm{CCI}$ co-treatment reverted the changes that $\mathrm{GC}$ evoked on different autophagy-related molecules (Fig. 7b). Specifically, GC + CCI-779 co-treatment increased LC3II with similar reverse changes in p62 protein levels (Fig. 7b). Additional confirmation was also obtained by LC3 staining, where $\mathrm{LC}^{+}$puncta in $\mathrm{GC}+\mathrm{CCI}$ treated cells were increased to control levels (Fig. 7c), suggesting that CCI779 blocked the GC-evoked impact of autophagic process. Moreover, CCI-779 treatment attenuated the GC-driven elevation of both exogenous (EGF-P301L-Tau) and endogenous (wild-type) Tau, as shown by both WB (Fig. 7e) and IF approaches (Fig. 7d), while it also reduced the levels of insoluble Tau aggregates (Fig. 7f); note that CCI alone had no impact on Tau levels (Supplementary Fig 3). Interestingly, CCI-779 blocked the GC-evoked elevation of different RBPs including TIA-1, G3BP, TLS/FUS and DDX5 

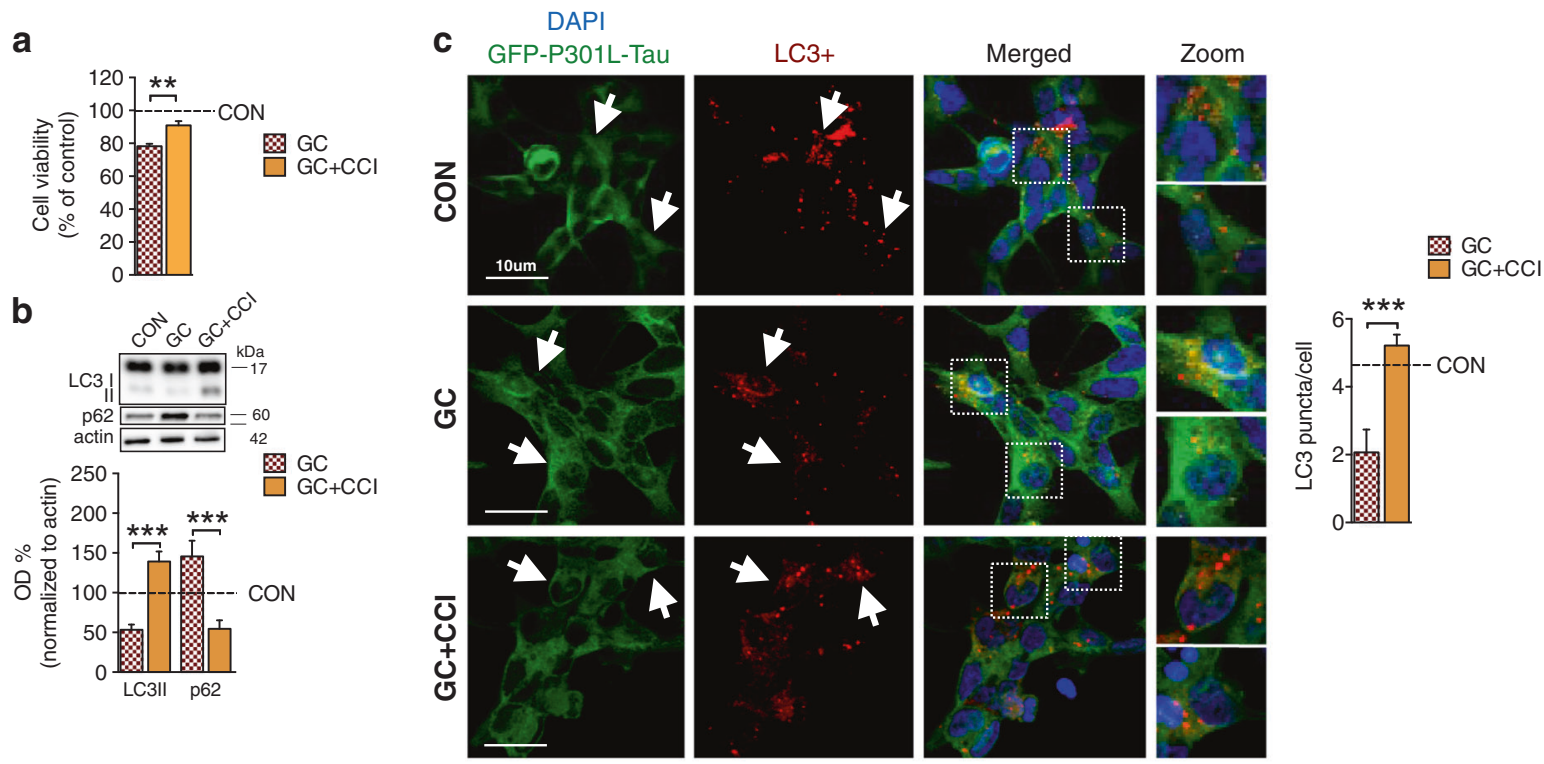

e
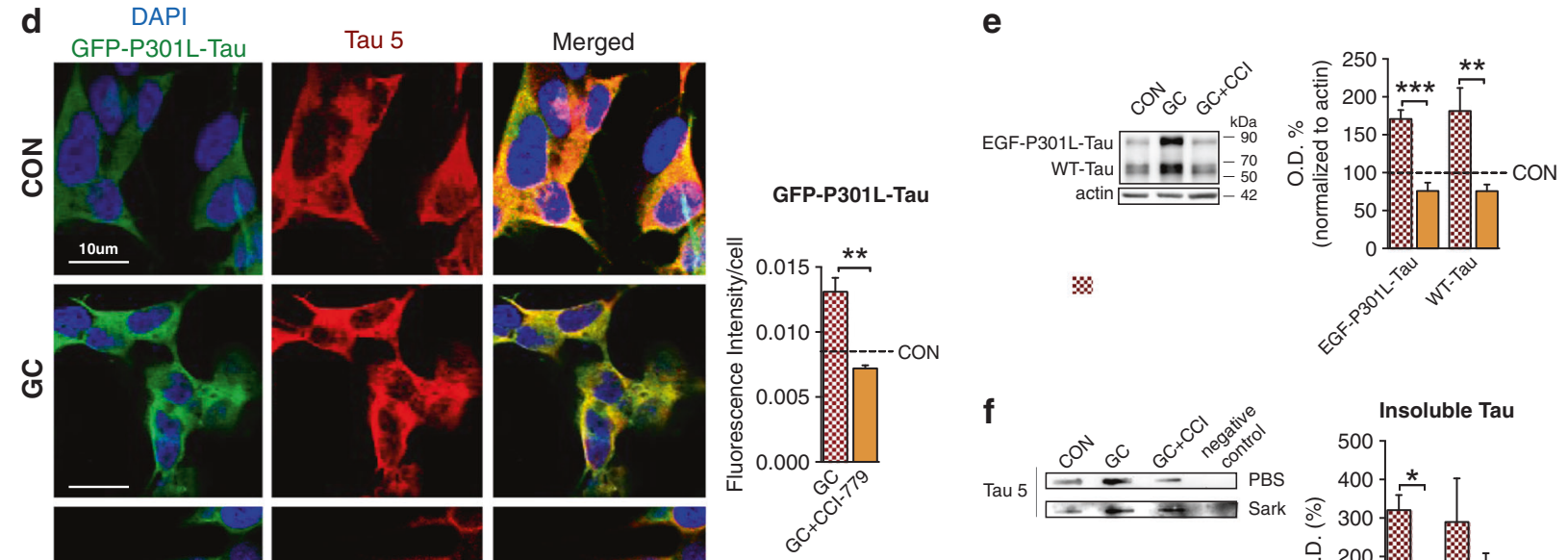

৩
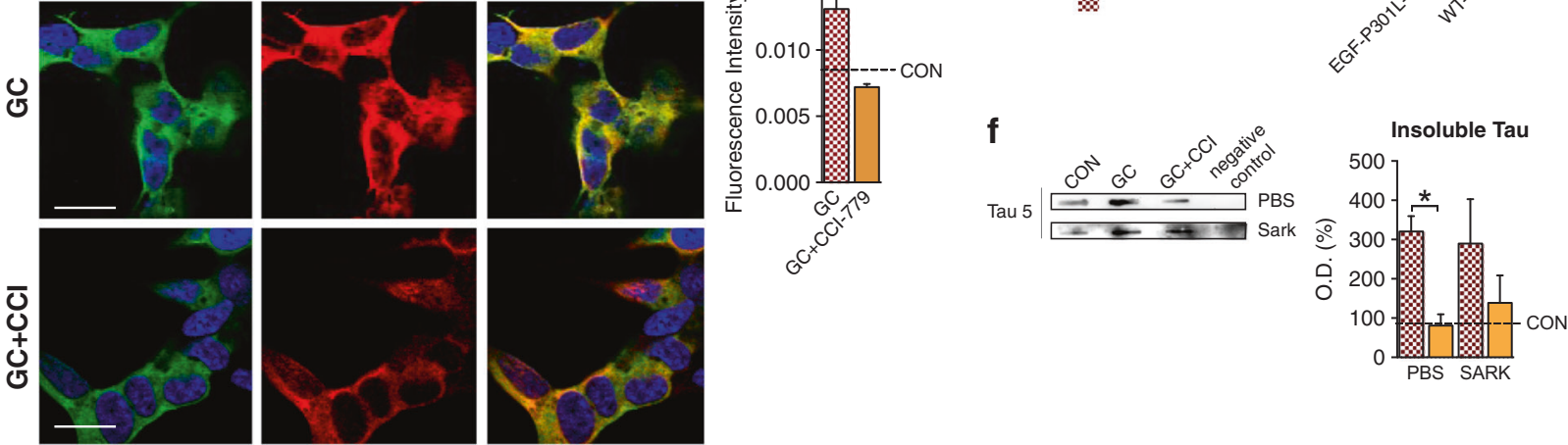

Fig. 7 mTOR-driven pharmacological stimulation of autophagy blocked GC-triggered Tau accumulation. a, b Combined treatment of GC with CCI-779 (Temsirolimus), a rapamycin analog, attenuated the GC-driven decrease of cell viability $(p=0.002)$ in EGF-P301LTau SH-SY5Y cells (a) and reversed the GC effect on autophagy markers, increasing protein levels of LC3II $(p<0.001)$ with parallel decrease of p62 levels $(p=0.001)(\mathbf{b})$. c IF staining of LC3 confirmed the CCI-

as assessed by WB (Fig. 8a) and IF analysis (Fig. 8b, c). Note that CCI-779 treatment blocked the GC-triggered cytoplasmic translocation and accumulation (in puncta) of the SGs core-nucleating protein TIA-1 preserving its localization to nucleus (Fig. 8b). These findings suggest that CCI-779-evoked mTOR inhibition can attenuate the GCevoked neurodegenerative cascades underlying Tau neurotoxicity.

779-evoked blockage of GC-driven reduction of LC3 puncta ( $p=$ 0.0012). d-f CCI-779 treatment reduced the GC-driven elevated IF levels of GFP-P301LTau $(p=0.002)(\mathbf{d})$; WB analysis revealed that CCI-779 attenuated the GC-driven accumulation of both EGF-P301LTau $(p<0.005)$ and WT-Tau $(p<0.001)$ levels $(\mathbf{e})$, also leading to decrease in insoluble Tau levels (f). All numeric data are represented mean \pm SEM, ${ }^{*} p<0.05 ;{ }^{* *} p<0.01 ;{ }^{* * *} p<0.0001$

\section{Discussion}

Understanding the molecular mechanisms that promote Tau misfolding and aggregation are of critical importance because of the key role played by Tau in mediating toxicity and neurodegeneration in $\mathrm{AD}[30,44]$. Although multiple preclinical and clinical trials have focused on Tau antiaggregation strategies with different compounds [45, 46], 
a
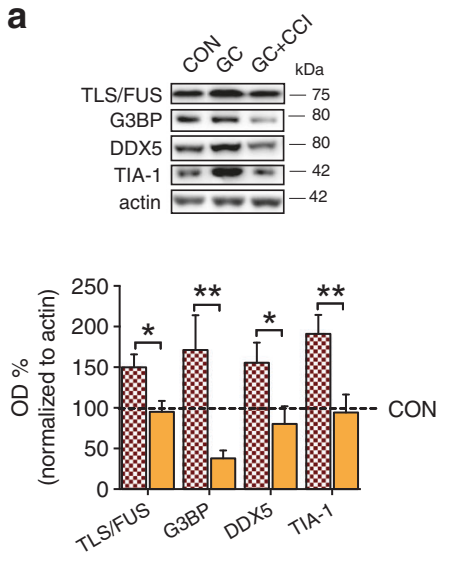

b $\begin{gathered}\text { DAPI } \\ \text { GFP-P301L-Tau }\end{gathered}$
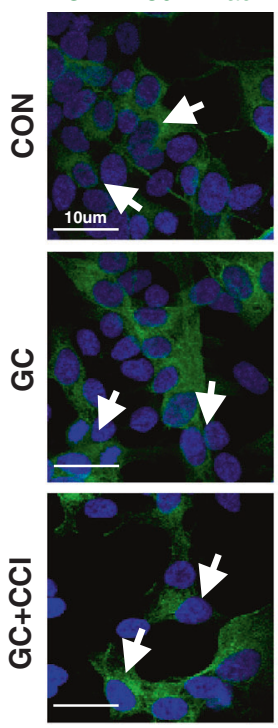

TIA-1
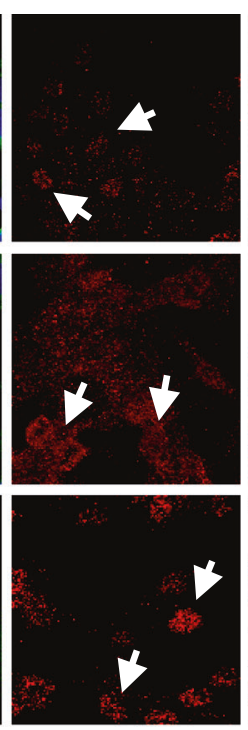

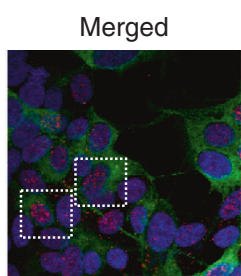

Zoom
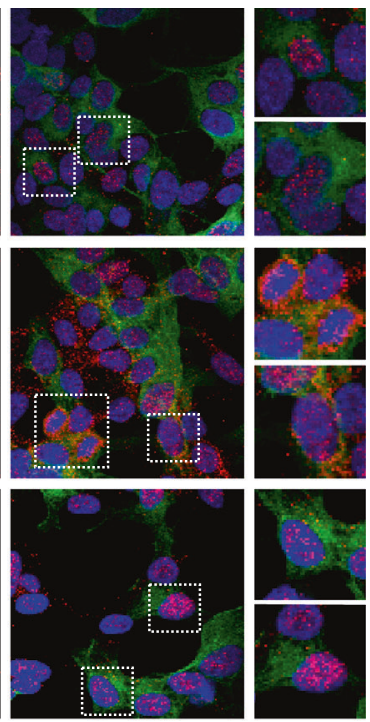

C

DAPI
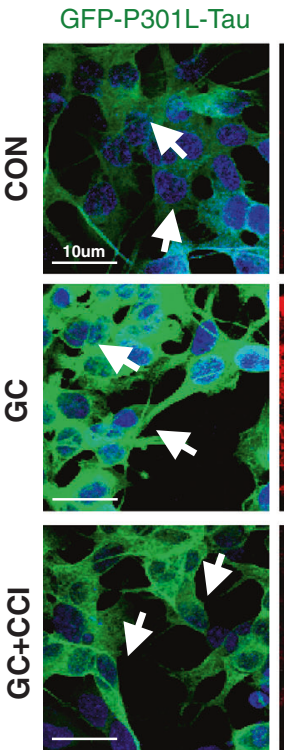

G3BP

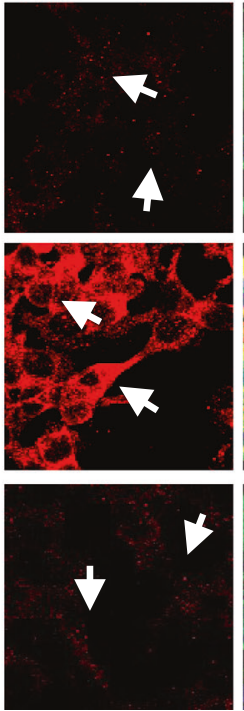

Merged
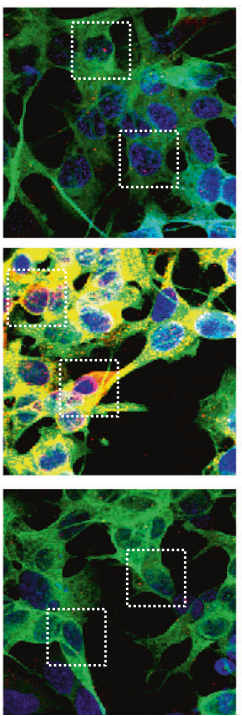

d Zoom
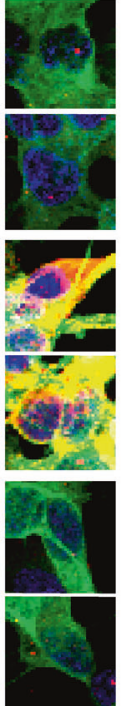

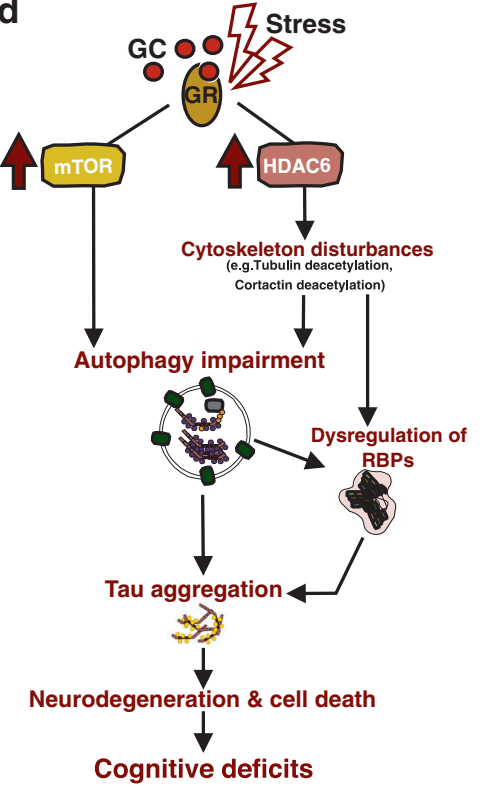

Fig. 8 The CCI-779 inhibitor of mTOR attenuated GC-induced dysregulation of RBPs. a-c GC-driven increase of different RBPs [TLS/ FUS G3BPand DDX5] was attenuated by co-treatment with CCI-779 ( $p=0.022 ; p=0.007 ; p=0.045$, respectively) (a); IF staining of TIA1 (b) and G3BP (c) confirmed the blockage of GC-driven induction of RBPs by CCI-779. c Working/hypothetical model integrating autophagy inhibition and dysregulation of RBPs in the cellular mechanisms through which chronic stress and/or high levels of stress hormones, glucocorticoids (GC), precipitate Tau pathology. Chronic exposure to environmental stress and/or prolonged signaling of GC receptors (GR) evoke the activation of mTOR signaling and the induction of Histone deacetylase 6 (HADC6) and subsequently, reduce acetylation of proteins related to cytoskeletal instability. These cellular events lead to inhibition of autophagic process that, together with the dysregulation and insolubilization of RBPs and the potential formation of SGs, may contribute to the accumulation of Tau and its neurotoxic aggregates causing cell death and cognitive deficits

between high cortisol levels and memory scores in $\mathrm{AD}$ subjects [5] and other implicate the activity of the HPA axis in the disease process [4]. Chronic stress is also correlated with earlier age of onset in familial AD [6], which further highlights a potential role of chronic stress and GC in the pathogenesis and/or progression of AD. Direct support of there remains a pressing need for new strategies to prevent disease progression in $\mathrm{AD}$.

The putative role of prolonged stress and excessive GC exposure in increasing brain vulnerability to disease pathology could have important implications for treatment of AD. Clinical studies reported a negative correlation 
the neurodegenerative potential of chronic stress is provided by multiple experimental studies, including ours, that show stress and GC trigger different parameters of Tau pathology such as aberrant hyperphosphorylation, somatodendritic accumulation [1, 7], and synaptic missorting [31, 32]. In addition, we have recently demonstrated that chronic stress also induces truncation and misfolding of Tau leading to the formation of neurotoxic Tau aggregates [8].

In the current study, we demonstrate that activation of the mTOR pathway is required for the effect of chronic stress and GC on Tau pathology, as the mTOR inhibitor (CCI-779) blocked the GC impact on Tau proteostasis (Fig. 8d). One of the main actions of mTOR inhibition is activation of autophagy. These results expand previous in vitro and in vivo observations showing that stress/GC reduced Tau turnover [9] and deregulates molecular chaperones responsible for Tau proteostasis [8]. Autophagy is thought to play a more important role than the proteosome in the catabolism of pathological Tau $[47,48]$ because the engulfing mechanism used by the autolysosomal system is more capable than the proteasome at degrading large macromolecular structures [10]. The potential role of autophagy is strongly supported by studies highlighting a specific relationship between autophagy deficits and Tau pathology in brains of $\mathrm{AD}$ patients and animals models of Tauopathies $[49,50]$.

Autophagy is a highly regulated process that is initiated by changes in phosphorylation states of individual components such as the Unc51-like-kinase complex, which is mainly regulated by mTOR. Interestingly, mTOR signaling is altered in AD [51, 52], with the levels of mTOR and its downstream targets, including p70S6K, being increased in AD human brains [13]. Accordingly, our current findings suggest that chronic stress and GC trigger mTOR activation and authophagic inhibition. We observed increased levels of phosphorylated mTOR and S6K, indicators of active mTOR signaling, as well as a reduced LC3II/LC3I ratio and an accumulation of $\mathrm{p} 62$. These findings suggest that chronic stress activates the mTOR pathway, which inhibits autophagy. The putative role of mTOR is supported by prior studies showing that chronic stress triggers mTOR activation in the hippocampus [53], and that mTOR activation is associated with increased total Tau levels in AD brains [54]. We further demonstrate that the use of the mTOR inhibitor, CCI-779, blocks the GC-driven Tau-related neurotoxicity and induction of aggregation-related cascades which is consistent with prior studies that show a decrease of mTOR signaling reverts Tau pathology $[27,55]$. These cumulative results point to a critical role for mTOR-dependent autophagy in the cellular mechanisms through which GC may trigger accumulation of Tau and its aggregates.

Recent studies from AD and FTDP human brains as well Tau Tg mice suggest that RBPs dysregulation and SGs represent a novel pathway that may contribute the development and progression of Tau pathology $[16,19]$. SGs are cytoplasmic complexes composed of phase separated complexes containing mRNAs and RBPs, which form to sequester nonessential transcripts in response to cellular stress. SGs are thought to constitute a protective mechanism against cellular stress by directing ribosomal system to translate mRNA transcripts coding for cytoprotective proteins [16]. The process of liquid-liquid phase separation and reversible aggregation that underlie SG formation is meant to be a transient event, but with prolonged SG induction is hypothesized to become pathological and neurotoxic [18, 19]. SGs have been suggested to accelerate Tau aggregation while, in a vicious cycle, Tau stimulates SG [19]. Factors known to stimulate Tau aggregation, including hyperphosphorylation and aggregation-prone mutations, also enhance the interactions between SGs and Tau [19].

The current study suggests a novel role for RBPs in the stress/GC-driven neuronal pathology. We demonstrate for the first time that chronic stress increases the cytosplasmic levels of different RBPs and SG-associated markers (e.g., TIA-1, PABP, G3BP, FUS, and DDX5) leading to its insoluble inclusion/accumulation. While SG biology involves the action of multiple different proteins leading to RBPs accumulation which is necessary for SGs [56-58], recent evidence demonstrates that TIA-1 pocess a primary role in the SG-related mechanism of Tau aggregation. Specifically, TIA-1 is transported from nucleus (where is predominantly found) to cytospasm where it interacts directly with Tau to stimulate its aggregation and insoluble accumulation/inclusion of other RBPs such as PABP and EWSR1 [17, 19, 59]. This is of particular importance as our current findings show that, under stressful conditions, TIA1 is traslocated from nucleus to cytosplam accompanied by insoluble accumulation of different SGs-associated proteins and Tau aggregates; future work should further study whether and how this RBP dysregulation and insolubilization correlates to altered SG formation. Furthermore, we have recently shown that Tau missorting and dendritic accumulation are also part of chronic stress/GC hippocampal pathology [31, 32], while missorting of Tau is hypothesized to facilitate formation of SGs as part of the translational stress response [19]. While the temporal profile and precise mechanisms underlying the stress/GC-evoked dysregulation of RBPs and SG-associated cascade remain to be elucidated, the working model suggested in this study (see Fig. 8d) opens a novel window of research and therapeutic exploration focusing on biology of RNA-protein interplay in stress-related pathologies.

The interface between autophagy and RBPs dysregulation and insolubilization that we highlight in this manuscript is supported by studies suggesting that inhibition of autophagy may impact in the dynamics and removal of SGs 
$[15,60]$; whether this occurs in the brain and in particular disease models was not previously known. The cytoskeletal machinery is known to facilitate the aggregation of RBPs to form SGs as well as contribute to the maturation of autophagic vesicles and autophagosome/lysosome fusion $[41,61]$. One of the molecules involved in these processes is histone deacetylase 6 (HDAC6), which, through the deacetylation of tubulin, reduces microtubule-dependent motility and thereby promotes the consolidation of cellular complexes such as SGs and autophagosomes [41, 42, 61]. Mounting evidence implicates HDAC6 in the formation of SGs in AD brain as HDAC6 seems to co-localize and interact with SG proteins under cellular stress; interestingly, HDAC6 is a SGs component interacting with G3BP [62, 63]. Additionally, pharmacological inhibition or genetic ablation of HDAC6 abolished SG formation [64], while the expression of HDAC6 significantly increases in the hippocampus and other brain regions of AD patients as well as in animal models of the disease [65, 66]. Our findings show that stress and GC increased HDAC6 levels, resulting in reduced acetylated levels of HDAC6's cytoskeletal targets, e.g., tubulin. Reduced acetylation of tubulin is associated with microtubule instability, which is also suggested to cause enlargement of SGs [67]. Interestingly, recent reports in mice show that HDAC6 inhibition increases resilience to stress $[68,69]$. While these findings point to HDAC6 as a potential regulator in the stress-driven Tau pathology (see Fig. 8d), future studies should clarify its specific role in different cellular pathways evoked by chronic stress and GC.

In conclusion, the current study adds to our limited knowledge about how chronic stress increases brain vulnerability to disease aiming to illuminate novel fundamental molecular mechanisms through which stress and GC may damage neuronal homeostasis precipitating Tau-driven neurodegeneration.

Acknowledgments We would like to thank Professor Juergen Gotz, (University of Queensland, Australia) for the kind offer of eGFPP301LTau SH-SY5Y cells and Dr. Bruno Almeida for his technical assistance. J.M.S. was granted with a PhD fellowship (SRFH/BD/ 88932/2012) by Portuguese Foundation for Science \& Technology (FCT); I.S. is holder of FCT Investigator grants (IF/01799/2013), C.D. is a recipient of $\mathrm{PhD}$ fellowship of $\mathrm{PHDoc}$ program and co-tutelle $\mathrm{PhD}$ student of UMinho-UPMC universities. This work was funded by FCT research grants "PTDC/SAU-NMC/113934/2009" (I.S.), the Portuguese North Regional Operational Program (ON.2) under the National Strategic Reference Framework (QREN), through the European Regional Development Fund (FEDER) as well as the Project Estratégico co-funded by FCT (PEst-C/SAU/LA0026/2013) and the European Regional Development Fund COMPETE (FCOMP-01-0124FEDER-037298) as well as the project NORTE-01-0145-FEDER000013, supported by the Northern Portugal Regional Operational Program (NORTE 2020), under the Portugal 2020 Partnership Agreement, through the European Regional Development Fund (FEDER). In addition, this work was partly funded by Canon Foundation in Europe.This work has been also funded by FEDER funds, through the Competitiveness Factors Operational Programme (COMPETE), and by National funds, through the Foundation for Science and Technology (FCT), under the scope of the project POCI-01-0145FEDER-007038. This study was also supported to BW by grants from NIH (AG050471, NS089544, and ES020395), the BrightFocus Foundation, the Alzheimer Association and the Cure Alzeimer Foundation. Human brain tissue was generously provided by the National Institute of Aging Boston University AD Center (P30AG13846)

\section{Compliance with ethical standards}

Conflict of interest B.W. is co-founder and chief scientific officer of Aquinnah Pharmaceutics Inc. The other authors declare that they have no conflict of interest.

\section{References}

1. Sotiropoulos I, Catania C, Pinto LG, Silva R, Pollerberg GE, Takashima A, et al. Stress acts cumulatively to precipitate Alzheimer's disease-like Tau pathology and cognitive deficits. J Neurosci. 2011;31:7840-7.

2. Sotiropoulos I, Cerqueira J, Catania C, Takashima A, Sousa N, Almeida O. Stress and glucocorticoid footprints in the brain-the path from depression to Alzheimer's disease. Neurosci Biobehav Rev. 2008;32:1161-73.

3. Launer LJ, Andersen K, Dewey ME, Letenneur L, Ott A, Amaducci LA, et al. Rates and risk factors for dementia and Alzheimer's disease: results from EURODEM pooled analyses. Neurology. 1999;52:78-84.

4. Hatzinger M, Z'Brun A, Hemmeter U, Seifritz E, Baumann F, Holsboer-Trachsler E, et al. Hypothalamic-pituitary-adrenal system function in patients with Alzheimer's disease. Neurobiol Aging. 1995;16:205-9.

5. Csernansky JG, Dong H, Fagan AM, Wang L, Xiong C, Holtzman $\mathrm{DM}$, et al. Plasma cortisol and progression of dementia in subjects with Alzheimer-type dementia. Am J Psychiatry. 2006;163: 2164-9.

6. Simard M, Hudon C, van Reekum R. Psychological distress and risk for dementia. Curr Psychiatry Rep. 2009;11:41-7.

7. Green KN, Billings LM, Roozendaal B, McGaugh JL, LaFerla FM. Glucocorticoids increase amyloid-beta and Tau pathology in a mouse model of Alzheimer's disease. J Neurosci. 2006;26:9047-56.

8. Sotiropoulos I, Silva J, Kimura T, Rodrigues AJ, Costa P, Almeida OFX, et al. Female hippocampus vulnerability to environmental stress as precipitating factor in Tau aggregation pathology. J Alzheimers Dis. 2014;40:1-12.

9. Sotiropoulos I, Catania C, Riedemann T, Fry J, Breen K, Michaelidis T, et al. Glucocorticoids trigger Alzheimer diseaselike pathobiochemistry in rat neuronal cells expressing human Tau. J Neurochem. 2008;107:385-97.

10. Boland B, Kumar A, Lee S, Platt FM, Wegiel J, Yu WH, et al. Autophagy induction and autophagosome clearance in neurons: relationship to autophagic pathology in Alzheimer's disease. J Neurosci. 2008;28:6926-37.

11. Nassif M, Hetz C. Autophagy impairment: a crossroad between neurodegeneration and Tauopathies. BMC Biol. 2012;10:78.

12. Mizushima N. The role of the Atg1/ULK1 complex in autophagy regulation. Curr Opin Cell Biol. 2010;22:132-9.

13. Caccamo A, Magri A, Medina DX, Wisely EV, Lopez-Aranda MF, Silva AJ, et al. mTOR regulates Tau phosphorylation and degradation: implications for Alzheimer's disease and other Tauopathies. Aging Cell. 2013;12:370-80. 
14. Vidal RL, Matus S, Bargsted L, Hetz C. Targeting autophagy in neurodegenerative diseases. Trends Pharmacol Sci. 2014;35:583-91.

15. Seguin SJ, Morelli FF, Vinet J, Amore D, De Biasi S, Poletti A, et al. Inhibition of autophagy, lysosome and VCP function impairs stress granule assembly. Cell Death Differ. 2014;21:1838-51.

16. Wolozin B. Regulated protein aggregation: stress granules and neurodegeneration. Mol Neurodegener. 2012;7:56.

17. Apicco DJ, Ash PEA, Maziuk B, Leblang C, Medalla M, Al Abdullatif A, et al. Reducing the RNA binding protein TIA1 protects against Tau-mediated neurodegeneration in vivo. Nat Neurosci. 2018;21:72-82.

18. Vanderweyde T, Yu H, Varnum M, Liu-Yesucevitz L, Citro A, Ikezu $\mathrm{T}$, et al. Contrasting pathology of the stress granule proteins TIA-1 and G3BP in Tauopathies. J Neurosci. 2012;32: 8270-83.

19. Vanderweyde T, Apicco DJ, Youmans-Kidder K, Ash PEA, Cook C, Lummertz da Rocha E, et al. Interaction of Tau with the RNAbinding protein TIA1 regulates Tau pathophysiology and toxicity. Cell Rep. 2016;15:1455-66.

20. Kimura T, Sahara N, Yamashita S, Murayama M, Mizoroki T, Yoshiike $\mathrm{Y}$, et al. Aggregation of detergent-insoluble Tau is involved in neuronal loss but not in synaptic loss. J Biol Chem. 2010;285:38692-386999.

21. Tanemura K, Murayama M, Akagi T, Hashikawa T, Tominaga T, Ichikawa M, et al. Neurodegeneration with Tau accumulation in a transgenic mouse expressing V337M human Tau. J Neurosci. 2002;22:133-41.

22. Kimura T, Fukuda T, Park JM, Murayama M, Mizoroki T, Yoshiike Y, Sahara N, Takashima AYS. Hyperphosphorylated Tau in parahippocampal cortex impairs place learning in aged mice expressing wild-type human Tau. EMBO J. 2007;26: 5143-52.

23. Sahara N, Lewis J, DeTure M, McGowan E, Dickson DW, Hutton $\mathrm{M}$, et al. Assembly of Tau in transgenic animals expressing P301L Tau: alteration of phosphorylation and solubility. J Neurochem. 2002;83:1498-508.

24. Bessa JM, Melo I, Marques F, Cerqueira JJ, Palha JA, Almeida OF, Sousa NFD. The mood-improving actions of antidepressants do not depend on neurogenesis but are associated with neuronal remodeling. Mol Psychiatry. 2009;14:764-73.

25. Sotiropoulos J, Kimura T, Rodirgues AJ, Costa P, ALmeida OFX, Sousa N, Takashima AIS. Female hippocampus vulnerability to environmental stress, a precipitating factor in Tau aggregation pathology. J Alzheimer's Dis. 2014;40:1-12.

26. Sun Y, Ip P, Chakrabartty A. Simple elimination of background fluorescence in formalin-fixed human brain tissue for immunofluorescence microscopy. J Vis Exp. 2017. https://doi.org/10. $3791 / 56188$.

27. Jiang T, Yu JT, Zhu XC, Zhang QQ, Cao L, Wang HF, et al. Temsirolimus attenuates tauopathy in vitro and in vivo by targeting Tau hyperphosphorylation and autophagic clearance. Neuropharmacology. 2014;85:121-30.

28. Korolchuk VI, Menzies FM, Rubinsztein DC. Mechanisms of cross-talk between the ubiquitin-proteasome and autophagylysosome systems. FEBS Lett. 2010;584:1393-8.

29. Giannakopoulos FR, Bussière T, Bouras C, Kövari E, Perl D, Morrison J, et al. Tangle and neuron numbers, but not amyloid load, predict cognitive status in Alzheimer's disease. Neurology. 2003;60:1495-1500.

30. Wang Y, Mandelkow E. Tau in physiology and pathology. Nat Rev Neurosci. 2015;17:22-35.

31. Lopes S, Vaz-Silva J, Pinto V, Dalla C, Kokras N, Bedenk B, et al. Tau protein is essential for stress-induced brain pathology. Proc Natl Acad Sci. 2016;113:E3755-E3763.
32. Pinheiro S, Silva J, Mota C, Vaz-Silva J, Veloso A, Pinto V, et al. Tau mislocation in glucocorticoid-triggered hippocampal pathology. Mol Neurobiol. 2015;53:4745-53.

33. Jost BC, Grossberg GT. The evolution of psychiatric symptoms in Alzheimer's disease: a natural history study. J Am Geriatr Soc. 1996;44:1078-81.

34. Wullschleger S, Loewith R, Hall MN. TOR signaling in growth and metabolism. Cell. 2006;124:471-84.

35. Wu X-N, Wang X-K, Wu S-Q, Lu J, Zheng M, Wang Y-H, et al. Phosphorylation of Raptor by p38beta participates in arseniteinduced mammalian target of rapamycin complex 1 (mTORC1) activation. J Biol Chem. 2011;286:31501-11.

36. Corrêa SAL, Eales KL. The role of p38 MAPK and its substrates in neuronal plasticity and neurodegenerative disease. J Signal Transduct. 2012;2012:1-12.

37. Alam J, Scheper W. Targeting neuronal MAPK14/p38 $\alpha$ activity to modulate autophagy in the Alzheimer disease brain. Autophagy. 2016;12:2516-20.

38. de Kloet ER, Joëls M, Holsboer F. Stress and the brain: from adaptation to disease. Nat Rev Neurosci. 2005;6:463-75.

39. Rissman M, Leea A, Justiceb N, Ricec K, Valed W, Staupa R, et al. Corticotropin-releasing factor receptor-dependent effects of repeated stress on Tau phosphorylation, solubility, and aggregation. Proc Natl Acad Sci. 2012;109:6277-82.

40. Liu-Yesucevitz L, Bilgutay A, Zhang YJ, Vanderwyde T, Citro A, Mehta T, et al. Tar DNA binding protein-43 (TDP-43) associates with stress granules: analysis of cultured cells and pathological brain tissue. PLoS ONE. 2010; 5. https://doi.org/10.1371/journal. pone.0013250.

41. Lee JY, Koga H, Kawaguchi Y, Tang W, Wong E, Gao YS, et al. HDAC6 controls autophagosome maturation essential for ubiquitin-selective quality-control autophagy. EMBO J. 2010;29: 969-80.

42. Lee JY, Nagano Y, Taylor JP, Lim KL, Yao TP. Disease-causing mutations in Parkin impair mitochondrial ubiquitination, aggregation, and HDAC6-dependent mitophagy. J Cell Biol. 2010; 189:671-9.

43. Malizzia LJ, Hsu A. Temsirolimus, an mTOR inhibitor for treatment of patients with advanced renal cell carcinoma. Clin J Oncol Nurs. 2008;12:639-46.

44. Ittner LM, Gotz J. Amyloid-beta and Tau-a toxic pas de deux in Alzheimer's disease. Nat Rev Neurosci. 2011;12:65-72.

45. Soeda Y, Yoshikawa M, Almeida OFX, Sumioka A, Maeda S, Osada $\mathrm{H}$, et al. ARTICLE toxic Tau oligomer formation blocked by capping of cysteine residues with 1,2-dihydroxybenzene groups. Nat Commun. 2015;6:1-12.

46. Novak P, Schmidt R, Kontsekova E, Zilka N, Kovacech B, Skrabana R, et al. Safety and immunogenicity of the Tau vaccine AADvac1 in patients with Alzheimer's disease: a randomised, double-blind, placebo-controlled, phase 1 trial. Lancet Neurol. 2016;4422:123-34.

47. Brown MR, Bondada V, Keller JN, Thorpe J, Geddes JW. Proteasome or calpain inhibition does not alter cellular Tau levels in neuroblastoma cells or primary neurons. J Alzheimers Dis. 2005; 7:15-24.

48. Feuillette S, Blard O, Lecourtois M, Frebourg T, Campion D, Dumanchin C. Tau is not normally degraded by the proteasome. J Neurosci Res. 2005;80:400-5.

49. Hara T, Nakamura K, Matsui M, Yamamoto A, Nakahara Y, Suzuki-Migishima R, et al. Suppression of basal autophagy in neural cells causes neurodegenerative disease in mice. Nature. 2006;441:885-9.

50. Ambegaokar SS, Jackson GR. The downward spiral of Tau and autolysosomes: a new hypothesis in neurodegeneration. Autophagy. 2012;8:1144-5. 
51. Caccamo A, Majumder S, Richardson A, Strong R, Oddo S. Molecular interplay between mammalian target of rapamycin (mTOR), amyloid-beta, and Tau: effects on cognitive impairments. J Biol Chem. 2010;285:13107-20.

52. Li I, Soininen H, Winblad B, Pei JXA. Levels of mTOR and its downstream targets 4E-BP1, eEF2, and eEF2 kinase in relationships with Tau in Alzheimer's disease brain. FEBS J. 2005;272:4211-20.

53. Polman JAE, Hunter RG, Speksnijder N, Van Den Oever JME, Korobko OB, McEwen BS, et al. Glucocorticoids modulate the mtor pathway in the hippocampus: differential effects depending on stress history. Endocrinology. 2012;153: 4317-27.

54. An WL, Cowburn RF, Li L, Braak H, Alafuzoff I, Iqbal K, et al. Up-regulation of phosphorylated/activated p70 S6 kinase and its relationship to neurofibrillary pathology in Alzheimer's disease. Am J Pathol. 2003;163:591-607.

55. Menzies J, Renna M, Bonin M, Riess O, Rubinsztein DCFMH. Autophagy induction reduces mutant ataxin-3 levels and toxicity in a mouse model of spinocerebellar ataxia type 3. Brain. 2010;133:93-104.

56. Kedersha NL, Gupta M, Li W, Miller I, Anderson P. RNAbinding proteins TIA-1 and TIAR link the phosphorylation of eIF2 alpha to the assembly of mammalian stress granules. J Cell Biol. 1999; 147:1431-41.

57. Gilkes N, Kedersha N, Ayodele M, Shen L, Stoecklin G, Dember LM, et al. Stress granule assembly is mediated by prion-like aggregation of TIA-1. Mol Biol Cell. 2004;15:5383-98.

58. Tourrière $\mathrm{H}$, Chebli $\mathrm{K}$, Zekri L, Courselaud B, Blanchard JM, Bertrand E, et al. The RasGAP-associated endoribonuclease G3BP assembles stress granules. J Cell Biol. 2003;160: 823-31.

59. Maziuk B, Ballance HI, Wolozin B. Dysregulation of RNA binding protein aggregation in neurodegenerative disorders. Front Mol Neurosci. 2017; 10. https://doi.org/10.3389/FNMOL.2017. 00089.
60. Buchan JR, Kolaitis RM, Taylor JP, Parker R XEukaryotic stress granules are cleared by autophagy and Cdc48/VCP function. Cell. 2013; 153. https://doi.org/10.1016/j.cell.2013.05.037.

61. Pandey UB, Nie Z, Batlevi Y, McCray BA, Ritson GP, Nedelsky $\mathrm{NB}$, et al. HDAC6 rescues neurodegeneration and provides an essential link between autophagy and the UPS. Nature. 2007;447: 859-63.

62. Kwon SH. The deacetylase HDAC6 is an essential component of stress granules and plays a critical role in the cellular response to stress. Genes Dev. 2007;21:3381-94.

63. Seigneurin-Berny D, Verdel A, Curtet S, Lemercier C, Garin J, Rousseaux $\mathrm{S}$, et al. Identification of components of the murine histone deacetylase 6 complex: link between acetylation and ubiquitination signaling pathways. Mol Cell Biol. 2001;21: 8035-44.

64. d'Ydewalle C, Bogaert E, Van Den Bosch L. HDAC6 at the Intersection of neuroprotection and neurodegeneration. Traffic. 2012;13:771-9.

65. Ding H, Dolan PJ, Johnson GV. Histone deacetylase 6 interacts with the microtubule-associated protein Tau. J Neurochem. 2008;106:2119-30.

66. Perez M, Santa-Maria I, Gomez de Barreda E, Zhu X, Cuadros R, Cabrero JR, et al. Tau-an inhibitor of deacetylase HDAC6 function. J Neurochem. 2009;109:1756-66.

67. Chernov KG, Barbet A, Hamon L, Ovchinnikov LP, Curmi PA, Pastr D. Role of microtubules in stress granule assembly: microtubule dynamical instability favors the formation of micrometric stress granules in cells. J Biol Chem. 2009;284:36569-80.

68. Espallergues J, Teegarden SL, Veerakumar A, Boulden J, Challis C, Jochems J, et al. HDAC6 regulates glucocorticoid receptor signaling in serotonin pathways with critical impact on stress resilience. J Neurosci. 2012;32:4400-16.

69. Jochems J, Boulden J, Lee BG, Blendy JA, Jarpe M, Mazitschek $\mathrm{R}$, et al. Antidepressant-like properties of novel HDAC6-selective inhibitors with improved brain bioavailability. Neuropsychopharmacology. 2014;39:389-400. 